\title{
Nucleon parton distributions in a light-front quark model
}

\author{
Thomas Gutsche $^{1}$, Valery E. Lyubovitskij ${ }^{1,2,3,4, a}$, Ivan Schmidt ${ }^{4}$ \\ ${ }^{1}$ Institut für Theoretische Physik, Kepler Center for Astro and Particle Physics, Universität Tübingen, Auf der Morgenstelle 14, 72076 Tübingen, \\ Germany \\ ${ }^{2}$ Department of Physics, Tomsk State University, Tomsk 634050, Russia \\ ${ }^{3}$ Laboratory of Particle Physics, Mathematical Physics Department, Tomsk Polytechnic University, Tomsk 634050, Russia \\ ${ }^{4}$ Departamento de Física y Centro Científico Tecnológico de Valparaíso (CCTVal), Universidad Técnica Federico Santa María, Casilla 110-V, \\ Valparaiso, Chile
}

Received: 14 October 2016 / Accepted: 26 January 2017 / Published online: 9 February 2017

(C) The Author(s) 2017. This article is published with open access at Springerlink.com

\begin{abstract}
Continuing our analysis of parton distributions in the nucleon, we extend our light-front quark model in order to obtain both the helicity-independent and the helicitydependent parton distributions, analytically matching the results of global fits at the initial scale $\mu \sim 1 \mathrm{GeV}$; they also contain the correct Dokshitzer-Gribov-Lipatov-AltarelliParisi evolution. We also calculate the transverse parton, Wigner and Husimi distributions from a unified point of view, using our light-front wave functions and expressing them in terms of the parton distributions $q_{v}(x)$ and $\delta q_{v}(x)$. Our results are very relevant for the current and future program of the COMPASS experiment at SPS (CERN).
\end{abstract}

\section{Introduction}

In Refs. [1,2] we proposed phenomenological light-front wave functions (LFWFs) for the nucleon, which produce a description of electromagnetic form factors of nucleons consistent with data and with the correct power behavior at higher scales $[3,4]$. The difference in the two papers [1,2] concerns the modeling of the $x$-dependence, which has an impact on the scaling behavior of nucleon parton distributions. In the first case [1] the nucleon parton distributions have the correct $x$ behavior at large scales, while at the initial scale $\mu \sim 1 \mathrm{GeV}$ they were different from the results of the world data analysis. In the second paper [2], we improved the $x$-dependence of the LFWFs in a such way that the modified LFWFs produced the correct helicity-independent parton distributions at the starting point for the Dokshitzer-Gribov-Lipatov-AltarelliParisi (DGLAP) evolution [5-8]. In the latter case [2] we also had some freedom in setting up the LFWFs, because we did not consider helicity-dependent parton distributions.

a e-mail: lubovit@tphys.physik.uni-tuebingen.de
A similar application of the pion LFWFs, resulting in good agreement with data and in the correct scaling of form factors and parton distributions, has been made in Ref. [9]. Note that the problem of constructing of the nucleon LFWFs was extensively studied in the literature starting from a pioneer paper by Brodsky et al. [10] and continuing by progress done by many groups in derivation of the LFWFs and its applications to nucleon phenomenology (see e.g. Refs. [11-27]).

In the present manuscript we derive the nucleon LFWFs where now the $x$-dependence is encoded by knowledge of the helicity-independent $q_{v}(x)$ and helicity-dependent $\delta q_{v}(x)$ valence parton distributions. The main advantage of our approach is that the derived LFWF does not depend on phenomenological parameters like masses of quark/diquark, which are not directly related to QCD. Restricting to zero current quark masses we obtain a reasonable description of data on nucleon form factors. The paper is organized as follows. In Sect. 2 we construct the nucleon LFWFs, which will be used for the calculation of parton distributions and form factors using the presentations of these quantities in terms of the LFWFs. In Sect. 3 we collect the well-known decompositions of the nucleon Dirac and Pauli form factors, parton distributions (including longitudinal, transverse, Wigner and Husimi distributions) in terms of the LFWFs. In Sect. 4 we present our numerical results and discussion. Finally, Sect. 5 contains our summary and conclusions. We have collected some technical material on the Wigner and Husimi parton distributions in the appendix.

\section{Nucleon light-front wave functions}

For simplicity we consider the quark-scalar diquark model, where the generic ansatz for the massless LFWFs at the initial scale $\mu_{0}=1 \mathrm{GeV}$ reads $[1,2]$ 
$\psi_{+q}^{+}\left(x, \mathbf{k}_{\perp}\right)=\varphi_{q}^{(1)}\left(x, \mathbf{k}_{\perp}\right)$,

$\psi_{-q}^{+}\left(x, \mathbf{k}_{\perp}\right)=-\frac{k^{1}+i k^{2}}{M_{N}} \varphi_{q}^{(2)}\left(x, \mathbf{k}_{\perp}\right)$,

$\psi_{+q}^{-}\left(x, \mathbf{k}_{\perp}\right)=\frac{k^{1}-i k^{2}}{M_{N}} \varphi_{q}^{(2)}\left(x, \mathbf{k}_{\perp}\right)$,

$\psi_{-q}^{-}\left(x, \mathbf{k}_{\perp}\right)=\varphi_{q}^{(1)}\left(x, \mathbf{k}_{\perp}\right)$,

where $\varphi_{q}^{(1)}$ and $\varphi_{q}^{(2)}$ are the LFWFs:

$$
\begin{aligned}
\varphi_{q}^{(1)}\left(x, \mathbf{k}_{\perp}\right)= & \frac{4 \pi}{M_{N}} \sqrt{\frac{q_{v}(x)+\delta q_{v}(x)}{2}} \sqrt{D_{q}^{(1)}(x)} \\
& \times \exp \left[-\frac{\mathbf{k}_{\perp}^{2}}{2 M_{N}^{2}} D_{q}^{(1)}(x)\right]
\end{aligned}
$$

$$
\begin{aligned}
\varphi_{q}^{(2)}\left(x, \mathbf{k}_{\perp}\right)= & \eta_{q} \frac{4 \pi}{M_{N}} \sqrt{\frac{q_{v}(x)-\delta q_{v}(x)}{2}} D_{q}^{(2)}(x) \\
& \times \exp \left[-\frac{\mathbf{k}_{\perp}^{2}}{2 M_{N}^{2}} D_{q}^{(2)}(x)\right] .
\end{aligned}
$$

Here $M_{N}$ is the nucleon mass, $q_{v}(x)$ and $\delta q_{v}(x)$ are the helicity-independent and helicity-dependent valence quark parton distributions (for these quantities the exact expressions from a world data analysis at the initial scale are understood), $D_{q}^{(1)}$ and $D_{q}^{(2)}$ are the longitudinal wave functions, connected to the electromagnetic form factors of the nucleon, $\eta_{u}=1$ and $\eta_{d}=-1$. Therefore, in our ansatz for the nucleon LFWFs only the functions $D_{q}^{(1)}(x)$ and $D_{q}^{(2)}(x)$ are free to be modeled. The parameterizations for $D_{q}^{(1)}(x)$ and $D_{q}^{(2)}(x)$ are not necessary the same and could be different, because these functions parametrize the LFWFs with different helicities of quark and nucleon (see below). Note that the nucleon mass $M_{N}$ is chosen as the scale parameter. It is easy to see that the choice of a specific scale $\Lambda$ in Eq. (1) is not important. A change of the scale $\Lambda \rightarrow \tilde{\Lambda}=\alpha \Lambda$ in (1) can be compensated for by a rescaling of the longitudinal functions $D_{q}^{(1)}$ and $D_{q}^{(2)}$ as

$D_{q}^{(i)} \rightarrow \tilde{D}_{q}^{(i)}=\alpha^{2} D_{q}^{(i)}$

For simplicity we therefore choose a scale coinciding with the nucleon mass $\Lambda=M_{N}$. Our functions $\varphi_{q}^{(1)}$ and $\varphi_{q}^{(2)}$ are normalized as

$$
\begin{array}{r}
\int \frac{\mathrm{d}^{2} \mathbf{k}_{\perp}}{16 \pi^{3}}\left[\varphi_{q}^{(1)}\left(x, \mathbf{k}_{\perp}\right)\right]^{2}=\frac{q_{v}(x)+\delta q_{v}(x)}{2}, \\
\int \frac{\mathrm{d}^{2} \mathbf{k}_{\perp}}{16 \pi^{3}} \frac{\mathbf{k}_{\perp}^{2}}{M_{N}^{2}}\left[\varphi_{q}^{(2)}\left(x, \mathbf{k}_{\perp}\right)\right]^{2}=\frac{q_{v}(x)-\delta q_{v}(x)}{2},
\end{array}
$$

and

$$
\begin{array}{r}
\int_{0}^{1} \mathrm{~d} x \int \frac{\mathrm{d}^{2} \mathbf{k}_{\perp}}{16 \pi^{3}}\left[\varphi_{q}^{(1)}\left(x, \mathbf{k}_{\perp}\right)\right]^{2}=\frac{n_{q}+g_{A}^{q}}{2}, \\
\int_{0}^{1} \mathrm{~d} x \int \frac{\mathrm{d}^{2} \mathbf{k}_{\perp}}{16 \pi^{3}} \frac{\mathbf{k}_{\perp}^{2}}{M_{N}^{2}}\left[\varphi_{q}^{(2)}\left(x, \mathbf{k}_{\perp}\right)\right]^{2}=\frac{n_{q}-g_{A}^{q}}{2},
\end{array}
$$

where $n_{q}$ is the number of $u$ or $d$ valence quarks in the proton and $g_{A}^{q}$ is the axial charge of a quark with flavor $q=u$ or $d$.

The functions $\varphi_{q}^{(1)}$ and $\varphi_{q}^{(2)}$ are generalizations of the LFWFs found by matching the electromagnetic form factors of the nucleon in soft-wall AdS/QCD [28-38] and lightfront QCD (see the detailed discussion in Refs. [1,2]). In particular, as a result of the matching procedure the functions $\varphi_{q}^{(i)}\left(x, \mathbf{k}_{\perp}\right)$ have been deduced:

$$
\begin{aligned}
\varphi_{q}^{\operatorname{AdS} / \mathrm{QCD}(i)}\left(x, \mathbf{k}_{\perp}\right) \sim & \frac{4 \pi}{\kappa} \frac{\sqrt{\log (1 / x)}}{1-x} \\
& \times \exp \left[-\frac{\mathbf{k}_{\perp}^{2}}{2 \kappa^{2}} \frac{\log (1 / x)}{(1-x)^{2}}\right] .
\end{aligned}
$$

Note that the derived LFWF is not symmetric under the exchange $x \rightarrow 1-x$. This asymmetry results from the matching of matrix elements of the bare electromagnetic current between the dressed LFWF in light-front QCD and of the dressed electromagnetic current between hadronic wave functions in AdS/QCD.

Concerning the $\mathbf{k}_{\perp}$ dependence of the $\varphi_{q}^{(1,2)}$ functions we use a specific functional form for them-Gaussian ansatz. However, a generalized ansatz for $\varphi_{q}^{(i)}$ reads

$$
\begin{aligned}
\varphi_{q}^{(1)}\left(x, \mathbf{k}_{\perp}\right)= & \frac{4 \pi}{M_{N}} \sqrt{\frac{q_{v}(x)+\delta q_{v}(x)}{2}} \sqrt{D_{q}^{(1)}(x)} \\
& \times \psi_{1}\left(-\frac{\mathbf{k}_{\perp}^{2}}{2 M_{N}^{2}} D_{q}^{(1)}(x)\right), \\
\varphi_{q}^{(2)}\left(x, \mathbf{k}_{\perp}\right)= & \eta_{q} \frac{4 \pi}{M_{N}} \sqrt{\frac{q_{v}(x)-\delta q_{v}(x)}{2}} D_{q}^{(2)}(x) \\
& \times \psi_{2}\left(-\frac{\mathbf{k}_{\perp}^{2}}{2 M_{N}^{2}} D_{q}^{(2)}(x)\right)
\end{aligned}
$$

where the functions $\psi_{1}$ and $\psi_{2}$ must satisfy the normalization conditions following from Eq. (5)

$$
\begin{aligned}
\int d^{2} \mathbf{k}_{\perp}\left[\psi_{1}\left(-\mathbf{k}_{\perp}^{2}\right)\right]^{2} & =\frac{\pi}{2}, \\
\int d^{2} \mathbf{k}_{\perp} \mathbf{k}_{\perp}^{2}\left[\psi_{2}\left(-\mathbf{k}_{\perp}^{2}\right)\right]^{2} & =\frac{\pi}{4} .
\end{aligned}
$$




\section{Light-front decompositions for the nucleon quantities}

\subsection{Form factors and parton distributions}

In this section we collect the well-known decompositions of the nucleon form factors and parton distributions in terms of the nucleon LFWFs. First we quote [39] the connection of the nucleon Dirac and Pauli form factors $F_{1,2}^{N}(N=p, n)$ with the valence quark distributions $F_{1,2}^{q}(q=u, d)$ in nucleons with

$$
F_{i}^{p(n)}\left(Q^{2}\right)=\frac{2}{3} F_{i}^{u(d)}\left(Q^{2}\right)-\frac{1}{3} F_{i}^{d(u)}\left(Q^{2}\right) .
$$

The valence quark distributions are related to the nucleon nonforward parton densities (NPDs) [39] $\mathcal{H}^{q}\left(x, Q^{2}\right)$ and $\mathcal{E}^{q}\left(x, Q^{2}\right)$ evaluated at zero skewness $\xi=0$ as

$$
\begin{aligned}
& F_{1}^{q}\left(Q^{2}\right)=\int_{0}^{1} \mathrm{~d} x \mathcal{H}^{q}\left(x, Q^{2}\right), \\
& F_{2}^{q}\left(Q^{2}\right)=\int_{0}^{1} \mathrm{~d} x \mathcal{E}^{q}\left(x, Q^{2}\right),
\end{aligned}
$$

where $Q^{2}=-q^{2}>0$ is the Euclidean momentum squared. At $Q^{2}=0$ the NPDs are related to the quark densitiesvalence $q_{v}(x)$ and magnetic $\mathcal{E}_{q}(x)$ as

$\mathcal{H}^{q}(x, 0)=q_{v}(x), \quad \mathcal{E}^{q}(x, 0)=\mathcal{E}^{q}(x)$,

which are normalized as

$n_{q}=F_{1}^{q}(0)=\int_{0}^{1} \mathrm{~d} x q_{v}(x)$,

$\kappa_{q}=F_{2}^{q}(0)=\int_{0}^{1} \mathrm{~d} x \mathcal{E}^{q}(x)$,

where $\kappa_{q}$ is the anomalous quark magnetic moment.

The nucleon Sachs form factors $G_{E / M}^{N}\left(Q^{2}\right)$ and the electromagnetic radii $\left\langle r_{E / M}^{2}\right\rangle^{N}$ are given in terms of the Dirac and Pauli form factors as

$$
\begin{aligned}
G_{E}^{N}\left(Q^{2}\right) & =F_{1}^{N}\left(Q^{2}\right)-\frac{Q^{2}}{4 m_{N}^{2}} F_{2}^{N}\left(Q^{2}\right), \\
G_{M}^{N}\left(Q^{2}\right) & =F_{1}^{N}\left(Q^{2}\right)+F_{2}^{N}\left(Q^{2}\right), \\
\left\langle r_{E}^{2}\right\rangle^{N} & =-\left.6 \frac{\mathrm{d} G_{E}^{N}\left(Q^{2}\right)}{\mathrm{d} Q^{2}}\right|_{Q^{2}=0}, \\
\left\langle r_{M}^{2}\right\rangle^{N} & =-\left.\frac{6}{G_{M}^{N}(0)} \frac{\mathrm{d} G_{M}^{N}\left(Q^{2}\right)}{\mathrm{d} Q^{2}}\right|_{Q^{2}=0},
\end{aligned}
$$

where $G_{M}^{N}(0) \equiv \mu_{N}$ is the nucleon magnetic moment.

The light-front representation $[11-13,40]$ for the Dirac and Pauli quark form factors is

$$
\begin{aligned}
F_{1}^{q}\left(Q^{2}\right)= & \int_{0}^{1} \mathrm{~d} x \int \frac{\mathrm{d}^{2} \mathbf{k}_{\perp}}{16 \pi^{3}}\left[\psi_{+q}^{+*}\left(x, \mathbf{k}_{\perp}^{\prime}\right) \psi_{+q}^{+}\left(x, \mathbf{k}_{\perp}\right)\right. \\
& \left.+\psi_{-q}^{+*}\left(x, \mathbf{k}_{\perp}^{\prime}\right) \psi_{-q}^{+}\left(x, \mathbf{k}_{\perp}\right)\right] \\
F_{2}^{q}\left(Q^{2}\right)= & -\frac{2 M_{N}}{q^{1}-i q^{2}} \int_{0}^{1} \mathrm{~d} x \int \frac{\mathrm{d}^{2} \mathbf{k}_{\perp}}{16 \pi^{3}} \\
& \times\left[\psi_{+q}^{+*}\left(x, \mathbf{k}_{\perp}^{\prime}\right) \psi_{+q}^{-}\left(x, \mathbf{k}_{\perp}\right)\right. \\
& \left.+\psi_{-q}^{+*}\left(x, \mathbf{k}_{\perp}^{\prime}\right) \psi_{-q}^{-}\left(x, \mathbf{k}_{\perp}\right)\right]
\end{aligned}
$$

where $\mathbf{k}_{\perp}^{\prime}=\mathbf{k}_{\perp}+\mathbf{q}_{\perp}(1-x)$. Here $\psi_{\lambda_{q} q}^{\lambda_{N}}\left(x, \mathbf{k}_{\perp}\right)$ are the LFWFs at the initial scale $\mu_{0}$ with specific helicities for the nucleon $\lambda_{N}= \pm$ and for the struck quark $\lambda_{q}= \pm$, where plus and minus correspond to $+\frac{1}{2}$ and $-\frac{1}{2}$, respectively. We work in the frame with $q=\left(0,0, \mathbf{q}_{\perp}\right)$, and where the Euclidean momentum squared is $Q^{2}=\mathbf{q}_{\perp}^{2}$. For the initial scale we choose the value $\mu_{0} \sim 1 \mathrm{GeV}$ which is used in the most of the global fits.

The expressions for the quark helicity-independent NPDs $\mathcal{H}^{q}$ and $\mathcal{E}^{q}$ in the nucleon read

$$
\begin{aligned}
\mathcal{H}^{q}\left(x, Q^{2}\right)= & \frac{q_{v}(x)+\delta q_{v}(x)}{2} e^{-t_{q}^{(11)}\left(x, Q^{2}\right)} \\
& +\frac{q_{v}(x)-\delta q_{v}(x)}{2} e^{-t_{q}^{(22)}\left(x, Q^{2}\right)} \\
& \times\left[1-t_{q}^{(22)}\left(x, Q^{2}\right)\right], \\
\mathcal{E}^{q}\left(x, Q^{2}\right)= & \mathcal{E}^{q}(x) e^{-t_{q}^{(12)}\left(x, Q^{2}\right)},
\end{aligned}
$$

where

$t_{q}^{(i j)}\left(x, Q^{2}\right)=\frac{Q^{2}}{4 M_{N}^{2}} \frac{2 D_{q}^{(i)}(x) D_{q}^{(j)}(x)}{D_{q}^{(i)}(x)+D_{q}^{(j)}(x)}(1-x)^{2}$.

The magnetization $\operatorname{PDF} \mathcal{E}^{q}(x)$ reads

$\mathcal{E}^{q}(x)=4 \eta_{q} \sqrt{q_{v}^{2}(x)-\delta q_{v}^{2}(x)} \sqrt{D_{q}^{(1)}(x)} \frac{(1-x) \sigma_{q}(x)}{\left[1+\sigma_{q}(x)\right]^{2}}$,

where $\sigma_{q}(x)=D_{q}^{(2)}(x) / D_{q}^{(1)}(x)$.

Our expressions for the helicity-independent NPDs and PDFs contain only the four unknown functions $D_{q}^{(i)}(x)$ with $q=u, d$ and $i=1,2$.

Note that by an appropriate choice of the longitudinal functions $D_{q}^{(i)}(x)$ we can guarantee the required scaling of the nucleon form factors at large $Q^{2}$. For example, if we adopt the following scaling of the quark helicity-independent PDFs:

$$
q_{v}(x) \sim(1-x)^{3}, \quad \mathcal{E}^{q}(x) \sim(1-x)^{5},
$$


we should choose the $D_{q}^{(i)}$ functions with the scaling behavior

$D_{q}^{(1)}(x) \sim(1-x)^{0}, \quad D_{q}^{(2)}(x) \sim(1-x)^{-1}$.

Thus we obtain the correct large $Q^{2}$ scaling of the quark form factors of the form

$F_{1}^{q}\left(Q^{2}\right) \sim \int_{0}^{1} \mathrm{~d} x(1-x)^{3} \exp \left[-\frac{Q^{2}}{4 M_{N}^{2}}(1-x)^{2}\right] \sim \frac{1}{Q^{4}}$

and

$F_{2}^{q}\left(Q^{2}\right) \sim \int_{0}^{1} \mathrm{~d} x(1-x)^{5} \exp \left[-\frac{Q^{2}}{4 M_{N}^{2}}(1-x)^{2}\right] \sim \frac{1}{Q^{6}}$.

This behavior guarantees the correct power scaling of the nucleon Dirac and Pauli form factors at higher $Q^{2}$ consistent with quark counting rules $[3,4]$ :

$F_{1}^{N}\left(Q^{2}\right) \sim 1 / Q^{4}, \quad F_{2}^{N}\left(Q^{2}\right) \sim 1 / Q^{6}$.

\subsection{Transverse momentum-dependent parton distributions}

In the quark-diquark model, the light-front decomposition for the transverse momentum-dependent parton distributions (TMDs) is discussed in detail in Ref. [17] (see also Ref. [26]). For recent progress in the extraction of TMDs from data, see e.g. Refs. [41-45]. The set of the valence quark $T$-even TMDs for the case of the quark-scalar diquark model is given by [17]:

$$
\begin{aligned}
f_{1}^{q_{v}}\left(x, \mathbf{k}_{\perp}\right) & \equiv h_{1 T}^{q_{v}}\left(x, \mathbf{k}_{\perp}\right) \\
& =\frac{1}{16 \pi^{3}}\left[\left|\psi_{+q}^{+}\left(x, \mathbf{k}_{\perp}\right)\right|^{2}+\left|\psi_{-q}^{+}\left(x, \mathbf{k}_{\perp}\right)\right|^{2}\right] \\
& =\frac{1}{16 \pi^{3}}\left[\left(\varphi_{q}^{(1)}\left(x, \mathbf{k}_{\perp}\right)\right)^{2}+\frac{\mathbf{k}_{\perp}^{2}}{M_{N}^{2}}\left(\varphi_{q}^{(2)}\left(x, \mathbf{k}_{\perp}\right)\right)^{2}\right],
\end{aligned}
$$

$$
\begin{aligned}
g_{1 L}^{q_{v}}\left(x, \mathbf{k}_{\perp}\right) & =\frac{1}{16 \pi^{3}}\left[\left|\psi_{+q}^{+}\left(x, \mathbf{k}_{\perp}\right)\right|^{2}-\left|\psi_{-q}^{+}\left(x, \mathbf{k}_{\perp}\right)\right|^{2}\right] \\
& =\frac{1}{16 \pi^{3}}\left[\left(\varphi_{q}^{(1)}\left(x, \mathbf{k}_{\perp}\right)\right)^{2}-\frac{\mathbf{k}_{\perp}^{2}}{M_{N}^{2}}\left(\varphi_{q}^{(2)}\left(x, \mathbf{k}_{\perp}\right)\right)^{2}\right],
\end{aligned}
$$

$$
\begin{aligned}
g_{1 T}^{q_{v}}\left(x, \mathbf{k}_{\perp}\right) \equiv & -h_{1 L}^{\perp q_{v}}\left(x, \mathbf{k}_{\perp}\right) \\
= & \frac{1}{16 \pi^{3}}\left[\psi_{+q}^{+*}\left(x, \mathbf{k}_{\perp}\right) \psi_{+q}^{-}\left(x, \mathbf{k}_{\perp}\right) \frac{M_{N}}{k^{1}-i k^{2}}\right. \\
& \left.+\psi_{+q}^{-*}\left(x, \mathbf{k}_{\perp}\right) \psi_{+q}^{+}\left(x, \mathbf{k}_{\perp}\right) \frac{M_{N}}{k^{1}+i k^{2}}\right] \\
= & \frac{1}{8 \pi^{3}} \varphi_{q}^{(1)}\left(x, \mathbf{k}_{\perp}\right) \varphi_{q}^{(2)}\left(x, \mathbf{k}_{\perp}\right),
\end{aligned}
$$

$$
\begin{aligned}
h_{1}^{q_{v}}\left(x, \mathbf{k}_{\perp}\right) & \equiv h_{1 T}^{q_{v}}\left(x, \mathbf{k}_{\perp}\right)+\frac{\mathbf{k}_{\perp}^{2}}{2 M_{N}^{2}} h_{1 T}^{\perp q_{v}}\left(x, \mathbf{k}_{\perp}\right) \\
& =\frac{1}{2}\left[f_{1}^{q_{v}}\left(x, \mathbf{k}_{\perp}\right)+g_{1 L}^{q_{v}}\left(x, \mathbf{k}_{\perp}\right)\right] \\
& =\frac{1}{16 \pi^{3}}\left|\psi_{+q}^{+}\left(x, \mathbf{k}_{\perp}\right)\right|^{2}=\frac{1}{16 \pi^{3}}\left(\varphi_{q}^{(1)}\left(x, \mathbf{k}_{\perp}\right)\right)^{2},
\end{aligned}
$$

$$
\begin{aligned}
\frac{\mathbf{k}_{\perp}^{2}}{2 M_{N}^{2}} h_{1 T}^{\perp q_{v}}\left(x, \mathbf{k}_{\perp}\right) & =\frac{1}{2}\left[g_{1 L}^{q_{v}}\left(x, \mathbf{k}_{\perp}\right)-f_{1}^{q_{v}}\left(x, \mathbf{k}_{\perp}\right)\right] \\
& =g_{1 L}^{q_{v}}\left(x, \mathbf{k}_{\perp}\right)-h_{1}^{q_{v}}\left(x, \mathbf{k}_{\perp}\right) \\
& =-\frac{1}{16 \pi^{3}}\left|\psi_{-q}^{+}\left(x, \mathbf{k}_{\perp}\right)\right|^{2} \\
& =-\frac{1}{16 \pi^{3}} \frac{\mathbf{k}_{\perp}^{2}}{M_{N}^{2}}\left(\varphi_{q}^{(2)}\left(x, \mathbf{k}_{\perp}\right)\right)^{2} .
\end{aligned}
$$

Using our expressions for the LFWFs we can express the TMDs through the PDFs

$$
\begin{aligned}
f_{1}^{q_{v}}\left(x, \mathbf{k}_{\perp}\right) & \equiv h_{1 T}^{q_{v}}\left(x, \mathbf{k}_{\perp}\right) \\
& =\mathcal{F}_{1}\left(x, \mathbf{k}_{\perp}\right)+\mathcal{F}_{2}\left(x, \mathbf{k}_{\perp}\right), \\
g_{1 L}^{q_{v}}\left(x, \mathbf{k}_{\perp}\right) & =\mathcal{F}_{1}\left(x, \mathbf{k}_{\perp}\right)-\mathcal{F}_{2}\left(x, \mathbf{k}_{\perp}\right), \\
g_{1 T}^{q_{v}}\left(x, \mathbf{k}_{\perp}\right) & \equiv-h_{1 L}^{\perp q_{v}}\left(x, \mathbf{k}_{\perp}\right)=\mathcal{F}_{3}\left(x, \mathbf{k}_{\perp}\right), \\
h_{1}^{q_{v}}\left(x, \mathbf{k}_{\perp}\right) & =\mathcal{F}_{1}\left(x, \mathbf{k}_{\perp}\right), \\
\frac{\mathbf{k}_{\perp}^{2}}{2 M_{N}^{2}} h_{1 T}^{\perp q_{v}}\left(x, \mathbf{k}_{\perp}\right) & =-\mathcal{F}_{2}\left(x, \mathbf{k}_{\perp}\right),
\end{aligned}
$$

where

$$
\begin{aligned}
\mathcal{F}_{1}\left(x, \mathbf{k}_{\perp}\right)= & \frac{1}{\pi M_{N}^{2}} \frac{q_{v}(x)+\delta q_{v}(x)}{2} D_{q}^{(1)}(x) e^{-\frac{\mathbf{k}_{\perp}^{2}}{M_{N}^{2}} D_{q}^{(1)}(x)}, \\
\mathcal{F}_{2}\left(x, \mathbf{k}_{\perp}\right)= & \frac{1}{\pi M_{N}^{2}} \frac{q_{v}(x)-\delta q_{v}(x)}{2} \frac{\mathbf{k}_{\perp}^{2}}{M_{N}^{2}}\left(D_{q}^{(2)}(x)\right)^{2} \\
& \times e^{-\frac{\mathbf{k}_{\perp}^{2}}{M_{N}^{2}} D_{q}^{(2)}(x)}, \\
\mathcal{F}_{3}\left(x, \mathbf{k}_{\perp}\right)= & \eta_{q} \sqrt{\frac{4 M_{N}^{2}}{\mathbf{k}_{\perp}^{2}} \mathcal{F}_{1}\left(x, \mathbf{k}_{\perp}\right) \mathcal{F}_{2}\left(x, \mathbf{k}_{\perp}\right)} \\
= & \frac{1}{\pi M_{N}^{2}} \eta_{q} \sqrt{q_{v}^{2}(x)-\delta q_{v}^{2}(x)} \sqrt{D_{q}^{(1)}(x)} D_{q}^{(2)}(x) \\
& \times e^{-\frac{\mathbf{k}_{\perp}^{2}}{2 M_{N}^{2}}}\left(D_{q}^{(1)}(x)+D_{q}^{(2)}(x)\right)
\end{aligned}
$$

Performing the $\mathbf{k}_{\perp}$-integration over the TMDs with

$$
\begin{aligned}
& \operatorname{TMD}(x)=\int \mathrm{d}^{2} \mathbf{k}_{\perp} \operatorname{TMD}\left(x, \mathbf{k}_{\perp}\right), \\
& \overline{\operatorname{TMD}}(x)=\int \mathrm{d}^{2} \mathbf{k}_{\perp} \frac{\mathbf{k}_{\perp}^{2}}{2 M_{N}^{2}} \operatorname{TMD}\left(x, \mathbf{k}_{\perp}\right),
\end{aligned}
$$


results in the identities

$$
\begin{aligned}
f_{1}^{q_{v}}(x) & \equiv h_{1 T}^{q_{v}}(x)=q_{v}(x), \\
g_{1 L}^{q_{v}}(x) & =\delta q_{v}(x), \\
g_{1 T}^{q_{v}}(x) & \equiv-h_{1 L}^{\perp q_{v}}(x)=\mathcal{E}^{q}(x) \frac{1+\sigma_{q}(x)}{2(1-x)}, \\
h_{1}^{q_{v}}(x) & =\frac{q_{v}(x)+\delta q_{v}(x)}{2}, \\
\overline{h_{1 T}^{\perp q_{v}}}(x) & =-\frac{q_{v}(x)-\delta q_{v}(x)}{2} .
\end{aligned}
$$

Finally, the integration over $x$ leads to the normalization conditions

$$
\begin{array}{r}
\int_{0}^{1} \mathrm{~d} x f_{1}^{q_{v}}(x)=\int_{0}^{1} \mathrm{~d} x h_{1 T}^{q_{v}}(x)=n_{q}, \\
\int_{0}^{1} \mathrm{~d} x g_{1 L}^{q_{v}}(x)=g_{A}^{q}, \\
\int_{0}^{1} \mathrm{~d} x h_{1}^{q_{v}}(x)=g_{T}^{q},
\end{array}
$$

where $g_{T}^{q}$ is the tensor charge.

Our TMD, independently on the longitudinal functions $D_{q}^{(i)}$, satisfy all relations and inequalities found before in theoretical approaches (see detailed discussion in Refs. [26, 46-49]. In particular, our TMDs in agreement with QCD and other models [48,49] (see also Ref. [26]) satisfy the following inequality relations:

$$
\begin{aligned}
& f_{1}^{q_{v}}\left(x, \mathbf{k}_{\perp}\right)>0, \\
& \left|g_{1 L}^{q_{v}}\left(x, \mathbf{k}_{\perp}\right)\right| \leq\left|f_{1}^{q_{v}}\left(x, \mathbf{k}_{\perp}\right)\right|, \\
& \left|h_{1}^{q_{v}}\left(x, \mathbf{k}_{\perp}\right)\right| \leq\left|f_{1}^{q_{v}}\left(x, \mathbf{k}_{\perp}\right)\right|, \\
& \left|g_{1 T}^{q_{v}}\left(x, \mathbf{k}_{\perp}\right)\right| \leq\left|F_{1}^{q_{v}}\left(x, \mathbf{k}_{\perp}\right)\right|=\sqrt{\frac{M_{N}^{2}}{\mathbf{k}_{\perp}^{2}}}\left|f_{1}^{q_{v}}\left(x, \mathbf{k}_{\perp}\right)\right|,
\end{aligned}
$$

which follow from the simple positivity condition for our functions $\mathcal{F}_{1}\left(x, \mathbf{k}_{\perp}\right)$ and $\mathcal{F}_{2}\left(x, \mathbf{k}_{\perp}\right)$ :

$$
\left[\mathcal{F}_{1}\left(x, \mathbf{k}_{\perp}\right)-\mathcal{F}_{2}\left(x, \mathbf{k}_{\perp}\right)\right]^{2} \geq 0 .
$$

Additionally, we confirm the inequality between the tensor and axial charges found in lattice QCD and different model (see discussion in Refs. $[26,50]$ ) and the generalized inequality

$\left|h_{1}^{q_{v}}\left(x, \mathbf{k}_{\perp}\right)\right| \geq\left|g_{1 L}^{q_{v}}\left(x, \mathbf{k}_{\perp}\right)\right|$

observed before in the framework of parton model [50] and derived recently in the quark-diquark model in Ref. [26]. Finally, our TMDs satisfy the non-linear relation found in Ref. [50] and recently confirmed in Ref. [26]: $h_{1}^{q_{v}}\left(x, \mathbf{k}_{\perp}\right) h_{1 T}^{\perp q_{v}}(x)=-\frac{1}{2}\left[h_{1 L}^{\perp q_{v}}(x)\right]^{2}$.

We would like to stress that the last inequality condition in Eq. (36) relating $g_{1 T}^{q_{v}}$ and $f_{1}^{q_{v}}$ after integration over $\mathbf{k}_{\perp}$ is also fulfilled in our approach. In particular, after integration over $\mathbf{k}_{\perp}$ we get

$$
\begin{aligned}
g_{1 T}^{q_{v}}(x)= & \mathcal{E}^{q}(x) \frac{1+\sigma_{q}(x)}{2(1-x)} \leq F_{1}^{q_{v}}(x)=\sqrt{\pi D_{q}^{(1)}(x)} \\
& \times\left[\frac{q_{v}(x)+\delta q_{v}(x)}{2}+\sigma_{q}(x) \frac{q_{v}(x)-\delta q_{v}(x)}{4}\right] .
\end{aligned}
$$

The inequality (40) is fulfilled because it is reduced to more trivial inequality

$$
\left[1+\sigma_{q}(x)\right]^{2}>\frac{8}{\pi} \sqrt{\sigma_{q}(x)},
$$

which occurs because of

$$
\left[1+\sigma_{q}(x)\right]^{2}>\sqrt{8 \sigma_{q}(x)}
$$

and

$$
\sqrt{8}>\frac{8}{\pi} \text {. }
$$

In Sect. 4 we present a plot where we compare our predictions for the $g_{1 T}^{q_{v}}(x)$ TMDs with corresponding upper limits defined by right-hand side of Eq. (40).

\subsection{Wigner distributions}

In light-front QCD the Wigner distributions read [27,51-54]

$$
\begin{aligned}
\rho^{q[\Gamma]}\left(x, \mathbf{b}_{\perp}, \mathbf{k}_{\perp} ; S\right)= & \int \frac{\mathrm{d}^{2} \Delta_{\perp}}{4 \pi^{2}} e^{-i \Delta_{\perp} \mathbf{b}_{\perp}} \\
& \times W^{q[\Gamma]}\left(x, \Delta_{\perp}, \mathbf{k}_{\perp} ; S\right),
\end{aligned}
$$

where $W^{q[\Gamma]}\left(x, \Delta_{\perp}, \mathbf{k}_{\perp} ; S\right)$ is the matrix element of the Wigner operator for $\Delta^{+}=0$ and $z^{+}=0$. The light-front decomposition of the Wigner matrix elements $W^{q[\Gamma]}\left(x, \Delta_{\perp}\right.$, $\left.\mathbf{k}_{\perp} ; S\right)$ is given by $[53,54]$

$$
\begin{aligned}
& W^{q\left[\gamma^{+}\right]}\left(x, \Delta_{\perp}, \mathbf{k}_{\perp} ; \pm e_{z}\right) \\
& \quad=\frac{1}{16 \pi^{3}}\left[\psi_{q+}^{ \pm \dagger}\left(x, \mathbf{k}_{\perp}^{+}\right) \psi_{q+}^{ \pm}\left(x, \mathbf{k}_{\perp}^{-}\right)+\psi_{q-}^{ \pm \dagger}\left(x, \mathbf{k}_{\perp}^{+}\right) \psi_{q-}^{ \pm}\left(x, \mathbf{k}_{\perp}^{-}\right)\right], \\
& W^{q\left[\gamma^{+} \gamma^{5}\right]}\left(x, \Delta_{\perp}, \mathbf{k}_{\perp} ; \pm e_{z}\right) \\
& \quad=\frac{1}{16 \pi^{3}}\left[\psi_{+q}^{ \pm \dagger}\left(x, \mathbf{k}_{\perp}^{+}\right) \psi_{+q}^{ \pm}\left(x, \mathbf{k}_{\perp}^{-}\right)-\psi_{-q}^{ \pm \dagger}\left(x, \mathbf{k}_{\perp}^{+}\right) \psi_{-q}^{ \pm}\left(x, \mathbf{k}_{\perp}^{-}\right)\right],
\end{aligned}
$$

where $\mathbf{k}_{\perp}^{ \pm}=\mathbf{k}_{\perp} \pm(1-x) \Delta_{\perp} / 2$.

Next we use the standard definitions of the Wigner distributions, specified by the nucleon helicity $\lambda_{N}$ and the quark helicity $\lambda_{q}[53,54]$ 


$$
\begin{aligned}
\rho_{\lambda_{N} \lambda_{q}}^{q}\left(x, \mathbf{b}_{\perp}, \mathbf{k}_{\perp}\right)= & \frac{1}{2}\left[\rho^{q\left[\gamma^{+}\right]}\left(x, \mathbf{b}_{\perp}, \mathbf{k}_{\perp}, \lambda_{N} \mathbf{e}_{z}\right)\right. \\
& \left.+\lambda_{q} \rho^{q\left[\gamma^{+} \gamma^{5}\right]}\left(x, \mathbf{b}_{\perp}, \mathbf{k}_{\perp}, \lambda_{N} \mathbf{e}_{z}\right)\right],
\end{aligned}
$$

which can be further expressed in terms of distributions where the proton and the struck quark are unpolarized $(U)$ or longitudinally polarized $(L)$ :

$$
\begin{aligned}
\rho_{\lambda_{N} \lambda_{q}}^{q} & =\frac{1}{2}\left[\rho_{U U}^{q}+\lambda_{N} \rho_{L U}^{q}+\lambda_{q} \rho_{U L}^{q}+\lambda_{N} \lambda_{q} \rho_{L L}^{q}\right], \\
\rho_{U U}^{q} & =\rho^{q\left[\gamma^{+}\right]}\left(\mathbf{e}_{z}\right)+\rho^{q\left[\gamma^{+}\right]}\left(-\mathbf{e}_{z}\right), \\
\rho_{L U}^{q} & =\rho^{q\left[\gamma^{+}\right]}\left(\mathbf{e}_{z}\right)-\rho^{q\left[\gamma^{+}\right]}\left(-\mathbf{e}_{z}\right), \\
\rho_{U L}^{q} & =\rho^{q\left[\gamma^{+} \gamma^{5}\right]}\left(\mathbf{e}_{z}\right)+\rho^{q\left[\gamma^{+} \gamma^{5}\right]}\left(-\mathbf{e}_{z}\right), \\
\rho_{L L}^{q} & =\rho^{q\left[\gamma^{+} \gamma^{5}\right]}\left(\mathbf{e}_{z}\right)-\rho^{q\left[\gamma^{+} \gamma^{5}\right]}\left(-\mathbf{e}_{z}\right) .
\end{aligned}
$$

The Fourier transforms $\omega_{A B}, A, B=U, L$ with respect to the $\mathbf{b}_{\perp}$ variable we define as

$\omega_{A B}^{q}\left(x, \Delta_{\perp}, \mathbf{k}_{\perp}\right)=\int \mathrm{d}^{2} \mathbf{b}_{\perp} e^{i \Delta_{\perp} \mathbf{b}_{\perp}} \rho_{A B}^{q}\left(x, \mathbf{b}_{\perp}, \mathbf{k}_{\perp}\right)$.

The expressions for the Wigner distributions $\rho_{A B}^{q}$ and $\omega_{A B}^{q}$ in the light-front quark-diquark approach are listed in Appendix A.

\subsection{Quark orbital angular momentum}

Following Ji [55] we define the quark contribution to the nucleon angular momentum:

$$
\begin{aligned}
J_{z}^{q} & =L_{z}^{q}+S_{z}^{q} \\
& =\frac{1}{2} \int_{0}^{1} \mathrm{~d} x x\left[\mathcal{H}^{q}(x, 0)+\mathcal{E}^{q}(x, 0)\right] \\
& =\frac{1}{2} \int_{0}^{1} \mathrm{~d} x x\left[q_{v}(x)+\mathcal{E}^{q}(x)\right],
\end{aligned}
$$

where the quark orbital angular momentum (OAM) $L_{z}^{q}$ and internal spin $S_{z}^{q}$ contributions are defined as

$$
\begin{aligned}
L_{z}^{q} & =\frac{1}{2} \int_{0}^{1} \mathrm{~d} x\left(x\left[\mathcal{H}^{q}(x, 0)+\mathcal{E}^{q}(x, 0)\right]-\tilde{H}^{q}(x, 0)\right) \\
& =\frac{1}{2} \int_{0}^{1} \mathrm{~d} x\left(x\left[q_{v}(x)+\mathcal{E}^{q}(x)\right]-\delta q_{v}(x)\right)
\end{aligned}
$$

and

$$
S_{z}^{q}=\frac{1}{2} \int_{0}^{1} \mathrm{~d} x \tilde{H}^{q}(x, 0)=\frac{1}{2} \int_{0}^{1} \delta q_{v}(x) .
$$

Integrating the TMD $-\frac{\mathbf{k}_{\perp}^{2}}{2 M_{N}^{2}} h_{1 T}^{\perp q_{v}}\left(x, \mathbf{k}_{\perp}\right)$ over $x$ and $\mathbf{k}_{\perp}$ one can derive the quantity $\mathcal{L}_{z}^{q}$,

$\mathcal{L}_{z}^{q}=-\int_{0}^{1} \mathrm{~d} x \int \mathrm{d}^{2} \mathbf{k}_{\perp} \frac{\mathbf{k}_{\perp}^{2}}{2 M_{N}^{2}} h_{1 T}^{\perp q_{v}}\left(x, \mathbf{k}_{\perp}\right)$

which is some quark models $[18,49,56,57]$ is equal to the quark OAM, but in general, in a gauge theory, it is not the case and $\mathcal{L}_{z}^{q} \neq L_{z}^{q}$ (see discussion in Refs. [21,58]). In particular, in our approach the quantity $\mathcal{L}_{z}^{q}$ is not related to the quark OAM $L_{z}^{q}$

$$
\begin{aligned}
\mathcal{L}_{z}^{q} & =\frac{1}{2} \int_{0}^{1} \mathrm{~d} x\left(q_{v}(x)-\delta q_{v}(x)\right)=\frac{n_{q}-g_{A}^{q}}{2} \\
& \neq L_{z}^{q} .
\end{aligned}
$$

Using $n_{u}=2$ and $n_{d}=1$ we get the relation between the quantities $\mathcal{L}_{z}^{q}$ and $S_{z}^{q}$ :

$\mathcal{L}_{z}^{u}=1-S_{z}^{u}, \mathcal{L}_{z}^{d}=\frac{1}{2}-S_{z}^{d}$

The next interesting quantity is the averaged quark orbital angular momentum (OAM) in a nucleon which is polarized in the $z$-direction $[21,22,53,54]$ :

$$
\begin{aligned}
l_{z}^{q} & =\left\langle\hat{L}_{z}^{q}\right\rangle^{\left[\gamma^{+}\right]}\left(\mathbf{e}_{z}\right) \\
& =\int_{0}^{1} \mathrm{~d} x \int \mathrm{d}^{2} \mathbf{k}_{\perp} \mathrm{d}^{2} \mathbf{b}_{\perp}\left(\mathbf{b}_{\perp} \times \mathbf{k}_{\perp}\right)_{z} \rho^{\left[\gamma^{+}\right]}\left(\mathbf{b}_{\perp}, \mathbf{k}_{\perp}, x, \mathbf{e}_{z}\right) \\
& =\int_{0}^{1} \mathrm{~d} x \int \mathrm{d}^{2} \mathbf{k}_{\perp} \mathrm{d}^{2} \mathbf{b}_{\perp}\left(\mathbf{b}_{\perp} \times \mathbf{k}_{\perp}\right)_{z} \rho_{L U}^{q}\left(\mathbf{b}_{\perp}, \mathbf{k}_{\perp}, x\right) \\
& =\frac{1}{2} \int_{0}^{1} \mathrm{~d} x(1-x)\left(q_{v}(x)-\delta q_{v}(x)\right) .
\end{aligned}
$$

One can see that the $l_{z}^{q}$ is related with TMD $h_{1 T}^{\perp q_{v}}\left(x, \mathbf{k}_{\perp}\right)$ by the integral representation over $x$ and $\mathbf{k}_{\perp}$ as

$l_{z}^{q}=-\int_{0}^{1} \mathrm{~d} x \int \mathrm{d}^{2} \mathbf{k}_{\perp} \frac{\mathbf{k}_{\perp}^{2}}{2 M_{N}^{2}}(1-x) h_{1 T}^{\perp q_{v}}\left(x, \mathbf{k}_{\perp}\right)$.

Another relevant quantity is the correlation between the quark spin and the orbital angular momentum $[21,22,53,54]$ with

$C_{z}^{q}=\int_{0}^{1} \mathrm{~d} x \int \mathrm{d}^{2} \mathbf{k}_{\perp} d^{2} \mathbf{b}_{\perp}\left(\mathbf{b}_{\perp} \times \mathbf{k}_{\perp}\right)_{z} \rho_{U L}^{q}\left(\mathbf{b}_{\perp}, \mathbf{k}_{\perp}, x\right)$, 
which in the quark-scalar diquark model [59] is opposite to the quantity $l_{z}^{q}$ with

$C_{z}^{q} \equiv-l_{z}^{q}$

because of $\rho_{U L}^{q}\left(\mathbf{b}_{\perp}, \mathbf{k}_{\perp}, x\right)=-\rho_{L U}^{q}\left(\mathbf{b}_{\perp}, \mathbf{k}_{\perp}, x\right)$. It is also confirmed in our calculations.

\subsection{Husimi distribution}

Finally, we consider the Husimi distribution function for the nucleon, which was recently discussed in detail in Refs. [60,61]. As was stressed in $[60,61]$ this distribution is better behaved and positive in comparison to the Wigner distribution. It also gives a probabilistic interpretation and can be used to define the entropy of the nucleon as a measure of the complexity of the partonic structure. It also could be connected to the color glass condensate approach at small $x$.

The Husimi distribution $h_{A B}^{q}\left(x, \mathbf{b}_{\perp}, \mathbf{k}_{\perp}\right)$ is defined as the integral of the Wigner distribution $\rho_{A B}^{q}\left(x, \mathbf{b}_{\perp}, \mathbf{k}_{\perp}\right)$ over the impact parameter $\mathbf{b}_{\perp}$ and the transverse momentum $\mathbf{k}_{\perp}$

$$
\begin{aligned}
h_{A B}^{q}\left(x, \mathbf{b}_{\perp}, \mathbf{k}_{\perp}\right)= & \frac{1}{\pi^{2}} \int \mathrm{d}^{2} \mathbf{k}_{\perp}^{\prime} \mathrm{d}^{2} \mathbf{b}_{\perp}^{\prime} e^{-\left(\mathbf{b}_{\perp}-\mathbf{b}_{\perp}^{\prime}\right)^{2} / l^{2}} \\
& \times e^{-l^{2}\left(\mathbf{k}_{\perp}-\mathbf{k}_{\perp}^{\prime}\right)^{2}} \rho_{A B}^{q}\left(x, \mathbf{b}_{\perp}^{\prime}, \mathbf{k}_{\perp}^{\prime}\right),
\end{aligned}
$$

where $1 / l^{2}=\left\langle\mathbf{k}_{\perp}^{2}\right\rangle$ is the average transverse momentum squared.

Note that the double moment of the Husimi distribution $h_{U U}^{q}$ and $h_{L L}^{q}$ is the ordinary PDF:

$$
\begin{aligned}
f_{1}^{q_{v}}(x) & =\int \mathrm{d}^{2} \mathbf{b}_{\perp} \mathrm{d}^{2} \mathbf{k}_{\perp} h_{U U}^{q}\left(x, \mathbf{b}_{\perp}, \mathbf{k}_{\perp}\right) \\
& =\int \mathrm{d}^{2} \mathbf{b}_{\perp} \mathrm{d}^{2} \mathbf{k}_{\perp} \rho_{U U}^{q}\left(x, \mathbf{b}_{\perp}, \mathbf{k}_{\perp}\right), \\
g_{1 L}^{q_{v}}(x) & =\int \mathrm{d}^{2} \mathbf{b}_{\perp} \mathrm{d}^{2} \mathbf{k}_{\perp} h_{L L}^{q}\left(x, \mathbf{b}_{\perp}, \mathbf{k}_{\perp}\right) \\
& =\int \mathrm{d}^{2} \mathbf{b}_{\perp} \mathrm{d}^{2} \mathbf{k}_{\perp} \rho_{L L}^{q}\left(x, \mathbf{b}_{\perp}, \mathbf{k}_{\perp}\right) .
\end{aligned}
$$

In the case of $h_{U L}^{q}$ and $h_{L U}^{q}$ the double moments equal zero.

In quantum mechanics the Husimi distribution $h_{\mathrm{QM}}$ is positive definite and one can define the so-called the HusimiWahrl entropy [62], which in our case can be extended to define the entropy of the nucleon $[60,61]$

$$
\begin{aligned}
S(x)= & -\int \mathrm{d}^{2} \mathbf{b}_{\perp} d^{2} \mathbf{k}_{\perp} h\left(x, \mathbf{b}_{\perp}, \mathbf{k}_{\perp}\right) \\
& \times \log \left[h\left(x, \mathbf{b}_{\perp}, \mathbf{k}_{\perp}\right)\right] .
\end{aligned}
$$

In particular, it is convenient to define the two quantities

$$
\begin{aligned}
S_{ \pm}^{q}(x)= & -\int \mathrm{d}^{2} \mathbf{b}_{\perp} \mathrm{d}^{2} \mathbf{k}_{\perp} h_{ \pm}^{q}\left(x, \mathbf{b}_{\perp}, \mathbf{k}_{\perp}\right) \\
& \times \log \left[h_{ \pm}^{q}\left(x, \mathbf{b}_{\perp}, \mathbf{k}_{\perp}\right)\right],
\end{aligned}
$$

where $h_{ \pm}^{q}=\left(h_{U U}^{q} \pm h_{L L}^{q}\right) / 2$. The expressions for $S_{ \pm}^{q}(x)$ are listed in Appendix A.

\section{Results}

In this paper we do not pretend to present a precise analysis of the available nucleon data. Instead we first would like to illustrate how our method works. For this purpose we use the results for the NLO helicity-independent and helicitydependent parton distributions at $\mu_{\mathrm{NLO}}^{2}=0.40 \mathrm{GeV}^{2}$ from Refs. [63] and [64] as input:

$$
\begin{aligned}
q(x) & =q_{v}(x)+\bar{q}(x), \delta q(x)=\delta q_{v}(x)+\delta \bar{q}(x), \\
x u_{v}(x) & =0.632 x^{0.43}(1-x)^{3.09}(1+18.2 x), \\
d_{v}(x) & =0.394(1-x) u_{v}(x), \\
x(\bar{u}+\bar{d})(x) & =1.24 x^{0.20}(1-x)^{8.5}(1-2.3 \sqrt{x}+5.7 x), \\
x(\bar{d}-\bar{u})(x) & =0.2 x^{0.43}(1-x)^{12.4}(1-13.3 \sqrt{x}+60 x), \\
x \delta u_{v}(x) & =2.043 x^{0.97}(1-x)^{0.64} u(x), \\
x \delta d_{v}(x) & =-2.709 x^{1.26}(1-x)^{1.06} d(x), \\
x \delta \bar{u}(x) & =1.727 x^{0.73}(1-x)^{2.00} \bar{u}(x), \\
\delta \bar{d}(x) & =\delta \bar{u}(x) \frac{\delta u(x)}{\delta d(x)} .
\end{aligned}
$$

The $D_{q}^{(i)}(x)$ are specified as

$$
\begin{aligned}
D_{q}^{(1)}(x) & =A_{q} \log (1 / x)(x+0.001)^{\alpha_{q}}(1-x)^{\beta_{q}}, \\
\sigma_{q}(x) & =N_{q} e^{-\gamma_{q} x} x^{\bar{\alpha}_{q}}(1-x)^{\bar{\beta}_{q}},
\end{aligned}
$$

where

$$
\begin{aligned}
& A_{u}=6.3385, A_{d}=1.17396, \\
& \alpha_{u}=0.37, \alpha_{d}=-0.31, \beta_{u}=0.09, \beta_{d}=-0.50, \\
& N_{u}=12.6, N_{d}=2.8, \gamma_{u}=3.70, \gamma_{d}=0.45, \\
& \bar{\alpha}_{u}=0.045, \bar{\alpha}_{d}=0, \bar{\beta}_{u}=-0.60, \bar{\beta}_{d}=0 .
\end{aligned}
$$

In Table 1 we present our results for the valence quark properties $\left(J_{z}^{q}, L_{z}^{q}, \mathcal{L}_{z}^{q}, l_{z}^{q}, C_{z}^{q}, \kappa_{q}\right)$ and compare them to the results of other calculations (light-cone constituent quark model (LCCQM) and chiral quark-soliton model ( $\chi$ QSM)) [21]. One can see that most of our results are different from the predictions of the LCCQM and $\chi \mathrm{QSM}$ approaches. This is caused by the difference in the magnetizazion PDFs $\mathcal{E}^{q}(x)$ (anomalous quark magnetic moments $\kappa_{q}$ ), helicitydependent PDFs $\delta q_{v}(x)$ (quark contributions to internal spin $\left.S_{z}^{q}\right)$. Note that our magnetization PDFs are consistent with data for nucleon electromagnetic form factors and helicitydependent PDFs $\delta q_{v}(x)$ are taken from Refs. [63,64]. Also we would like to stress that our results for the quantities $\mathcal{L}_{z}^{q}$ are clearly understood because they are related to the quantities $S_{z}^{q}$ by the relations (54). 
Table 1 Valence quark properties

\begin{tabular}{lrrr}
\hline Quantity & LCCQM [21] & $\chi$ QCM [21] & \multicolumn{1}{c}{ Our } \\
\hline$J_{z}^{u}$ & 0.569 & 0.566 & 0.358 \\
$J_{z}^{d}$ & -0.069 & -0.066 & -0.010 \\
$L_{z}^{u}$ & 0.071 & -0.008 & 0.055 \\
$L_{z}^{d}$ & 0.055 & 0.077 & -0.001 \\
$S_{z}^{u}$ & 0.498 & 0.574 & 0.303 \\
$S_{z}^{d}$ & -0.124 & -0.143 & -0.009 \\
$\mathcal{L}_{z}^{u}$ & 0.169 & 0.093 & 0.697 \\
$\mathcal{L}_{z}^{d}$ & -0.042 & -0.023 & 0.509 \\
$l_{z}^{u}$ & 0.131 & 0.073 & 0.598 \\
$l_{z}^{d}$ & -0.005 & -0.004 & 0.404 \\
$C_{z}^{u}$ & 0.227 & 0.130 & -0.598 \\
$C_{z}^{d}$ & 0.187 & 0.109 & -0.404 \\
$\kappa_{u}$ & 1.867 & 1.766 & 1.673 \\
$\kappa_{d}$ & -1.579 & -1.551 & -2.033 \\
\hline
\end{tabular}

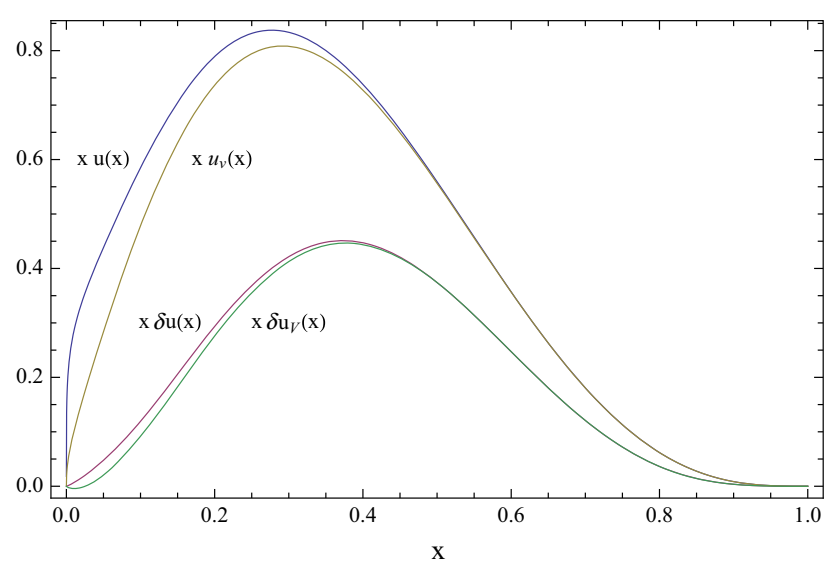

Fig. $1 u$ Quark PDFs multiplied with $x$

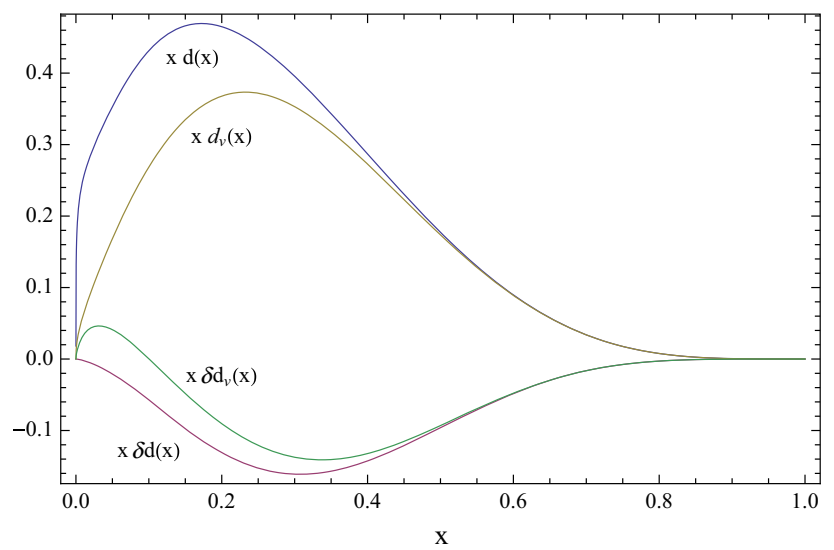

Fig. $2 d$ Quark PDFs multiplied with $x$

In Figs. 1, 2, 3, 4, 5, 6, 7, 8, 9, 10, 11, 12, 13, 14, 15, 16, 17 , $18,19,20,21$, and 22 we plot the results for the $x$-dependence of the unpolarized and polarized PDFs, TMDs, Wigner and

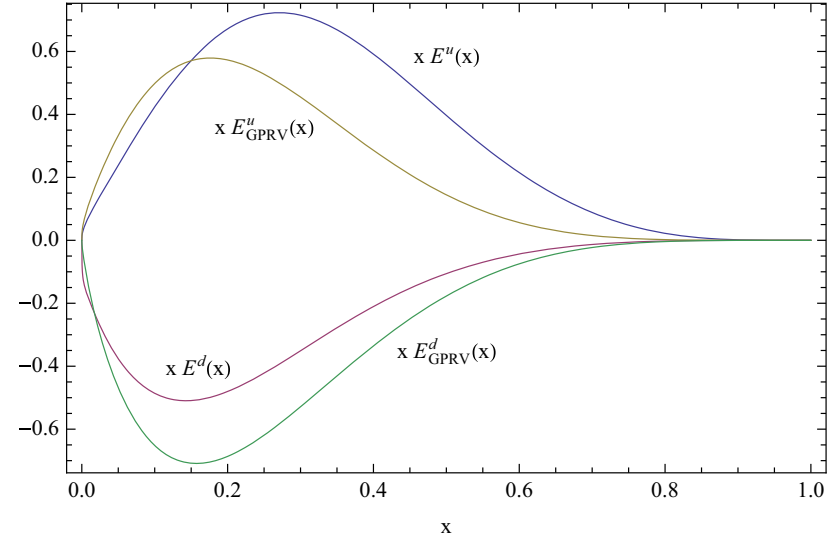

Fig. 3 Quark magnetization PDFs: $x \mathcal{E}^{q}$ - our results, $x \mathcal{E}_{G P R V}^{q}$ - results of Ref. [68]

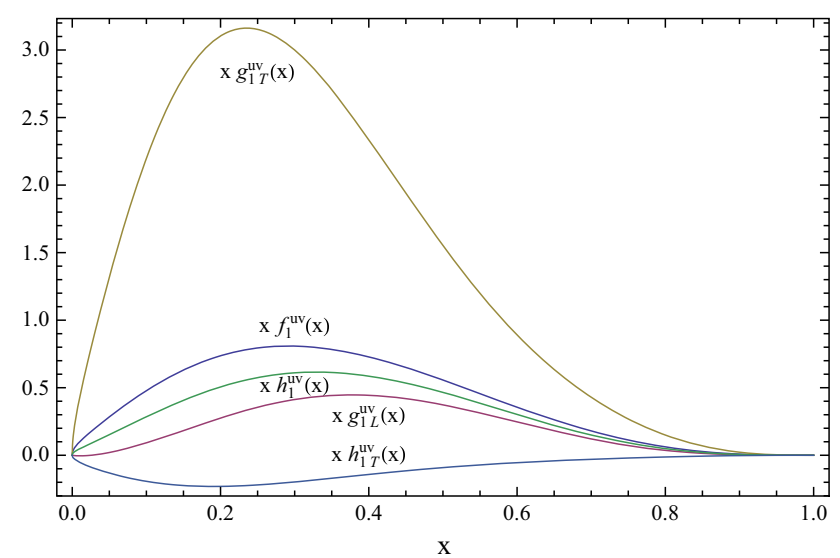

Fig. $4 u$ Quark TMDs multiplied with $x$

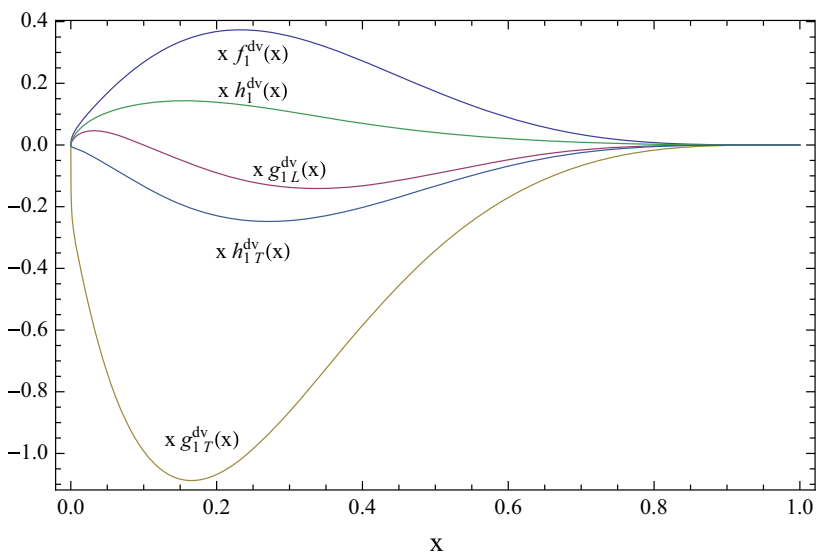

Fig. $5 d$ Quark TMDs multiplied with $x$

Husimi distributions, and we indicate selected results for the quark and nucleon electromagnetic form factors. The data on the quark decomposition of the nucleon form factors are taken from Refs. [65-67]. In particular, in Fig. 3 we show our predictions for magnetization $\mathrm{PDFs} \mathcal{E}^{q}$ and compare them 


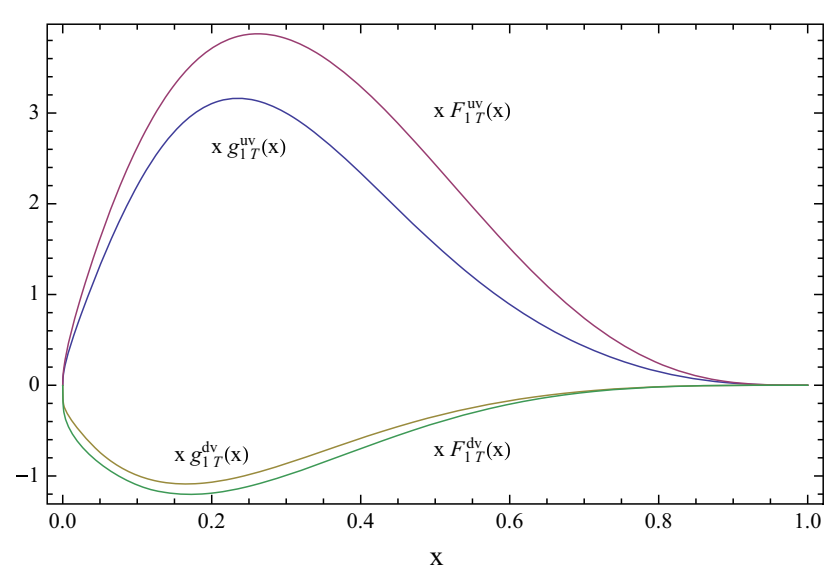

Fig. 6 Comparison our predictions for $x g_{1 T}^{q_{v}}(x)$ quark TMDs multiplied with corresponding upper limits $x F_{1}^{q_{v}}(x)$

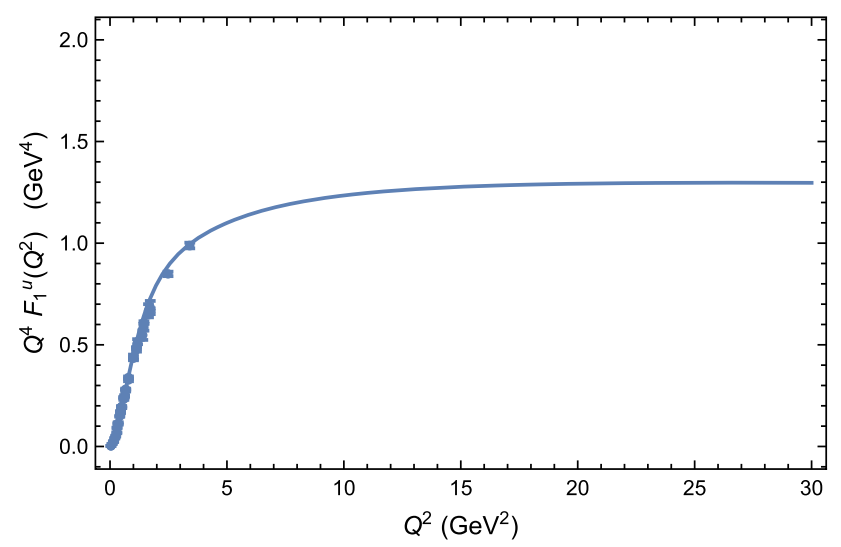

Fig. 7 Dirac $u$ quark form factor multiplied by $Q^{4}$

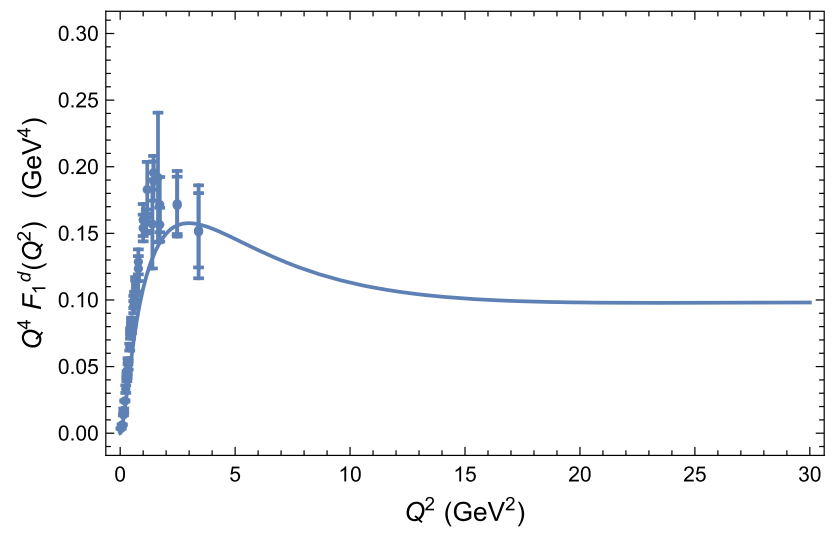

Fig. 8 Dirac $d$ quark form factor multiplied by $Q^{4}$

with results of Ref. [68]. In Fig. 6 we present a comparison of our predictions for $x g_{1 T}^{q_{v}}(x)$ quark TMDs with the corresponding upper limits $x F_{1}^{q_{v}}(x)$. One can see that our results for $g_{1 T}^{q_{v}}(x)$ are consistent with model-independent inequalities derived in Ref. [48]. Note that before in Sect. 3 we

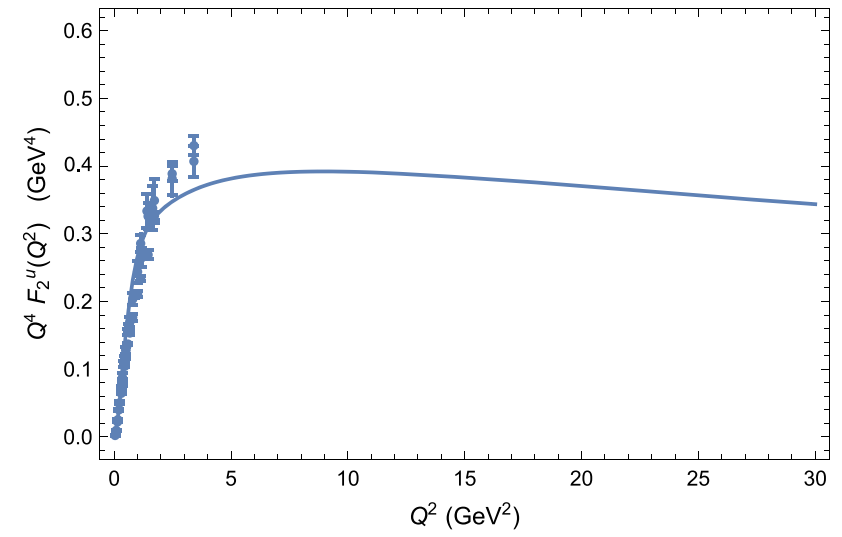

Fig. 9 Pauli $u$ quark form factor multiplied by $Q^{4}$

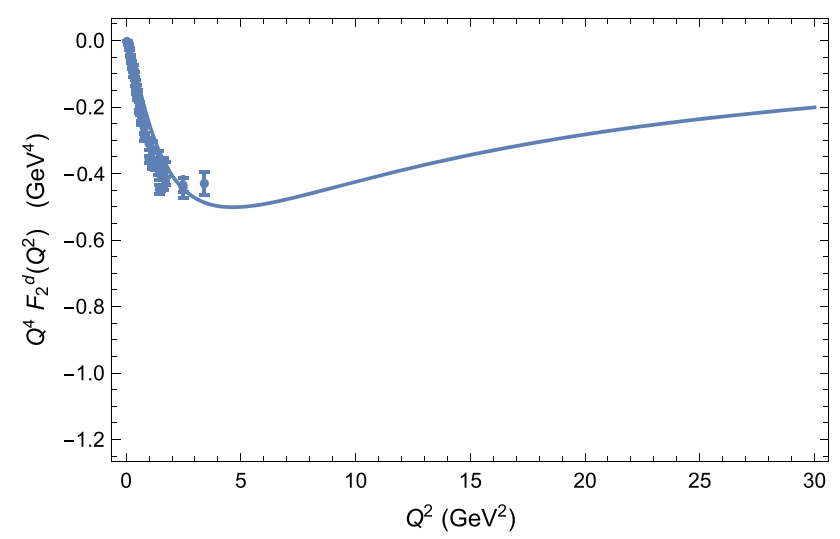

Fig. 10 Pauli $d$ quark form factor multiplied by $Q^{4}$

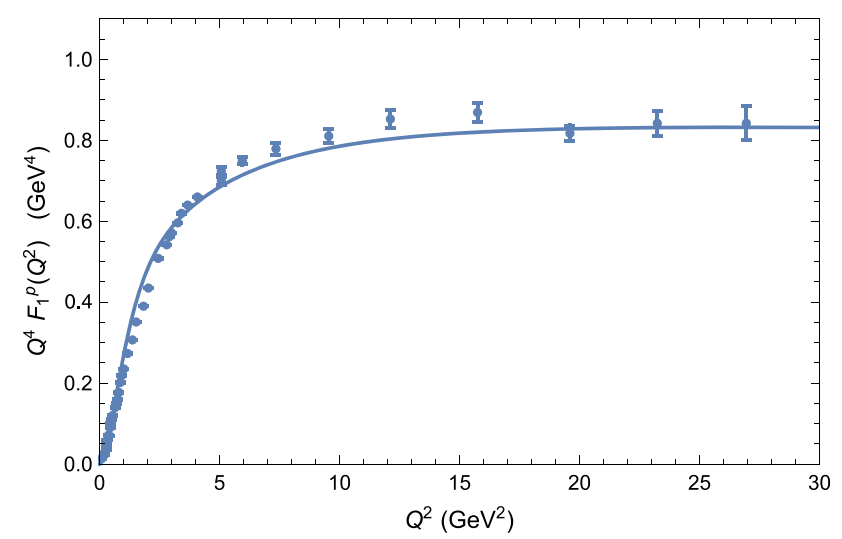

Fig. 11 Dirac proton form factor multiplied by $Q^{4}$

proved it analytically. Our Wigner distributions are negative for longitudinal-logitudinal polarized case of the $d$-quark and for unpolarized-longitudinal polarized case for both quark flavors. Note that negative Wigner distributions have been obtained in some approaches, e.g. after including the gluons (see discussion in Refs. [60,61,69]). 


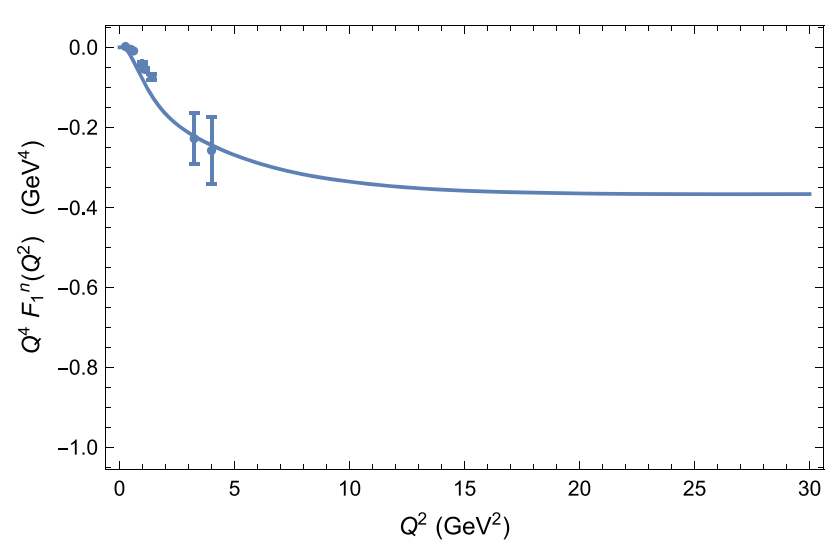

Fig. 12 Dirac neutron form factor multiplied by $Q^{4}$

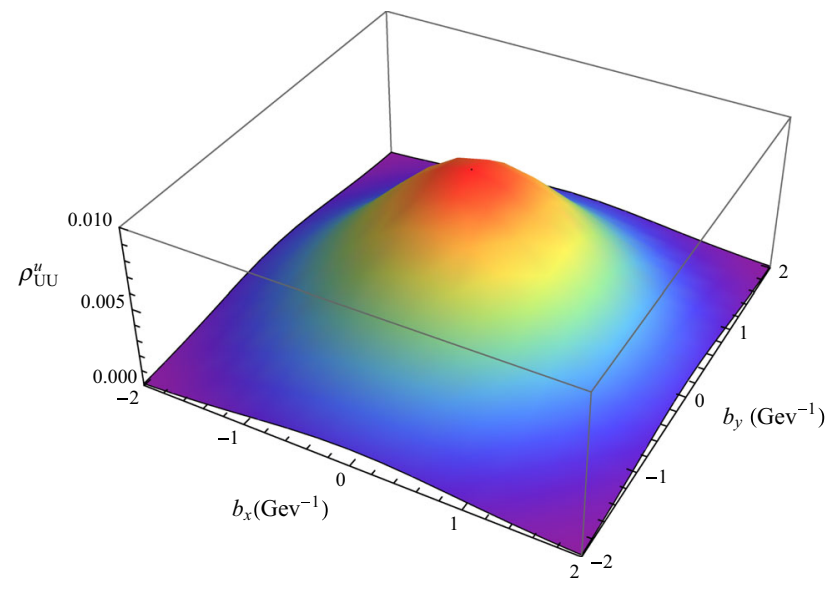

Fig. 13 Wigner distribution $\rho_{U U}^{u}\left(x, \mathbf{b}_{\perp}, \mathbf{k}_{\perp}\right)$ at $x=0.5, k_{x}=k_{y}=$ $0.5 \mathrm{GeV}$

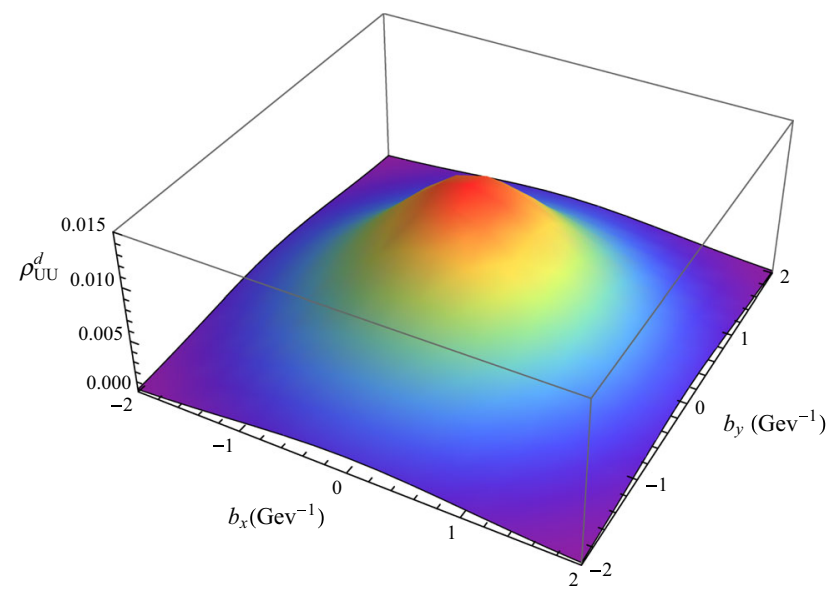

Fig. 14 Wigner distribution $\rho_{U U}^{d}\left(x, \mathbf{b}_{\perp}, \mathbf{k}_{\perp}\right)$ at $x=0.5, k_{x}=k_{y}=$ $0.5 \mathrm{GeV}$

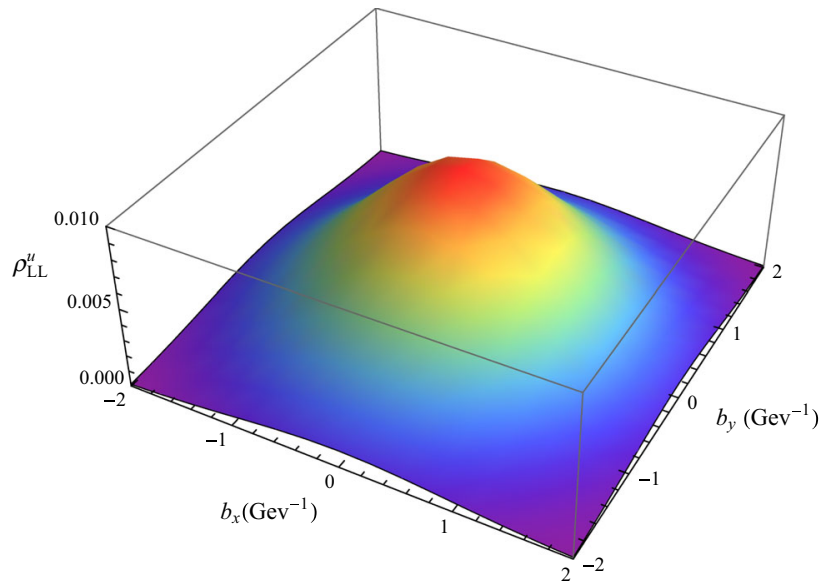

Fig. 15 Wigner distribution $\rho_{L L}^{u}\left(x, \mathbf{b}_{\perp}, \mathbf{k}_{\perp}\right)$ at $x=0.5, k_{x}=k_{y}=$ $0.5 \mathrm{GeV}$

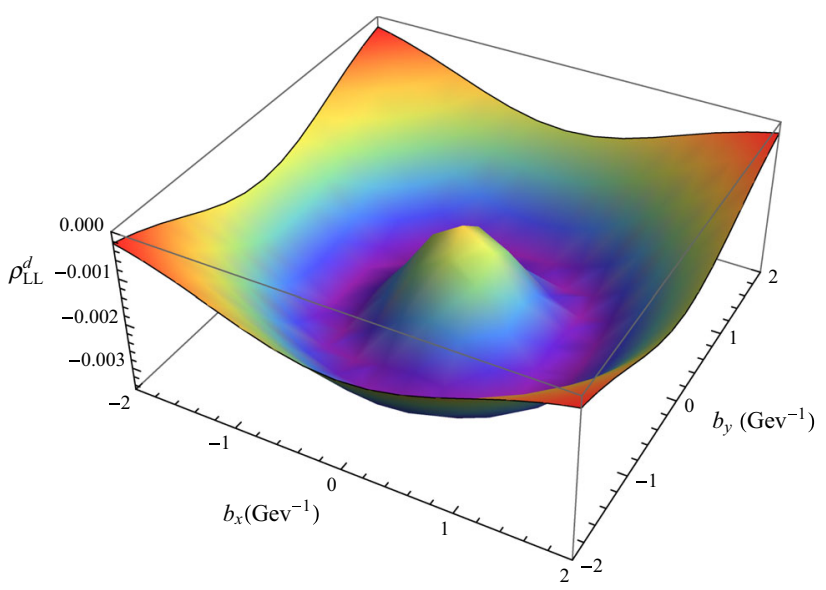

Fig. 16 Wigner distribution $\rho_{L L}^{d}\left(x, \mathbf{b}_{\perp}, \mathbf{k}_{\perp}\right)$ at $x=0.5, k_{x}=k_{y}=$ $0.5 \mathrm{GeV}$

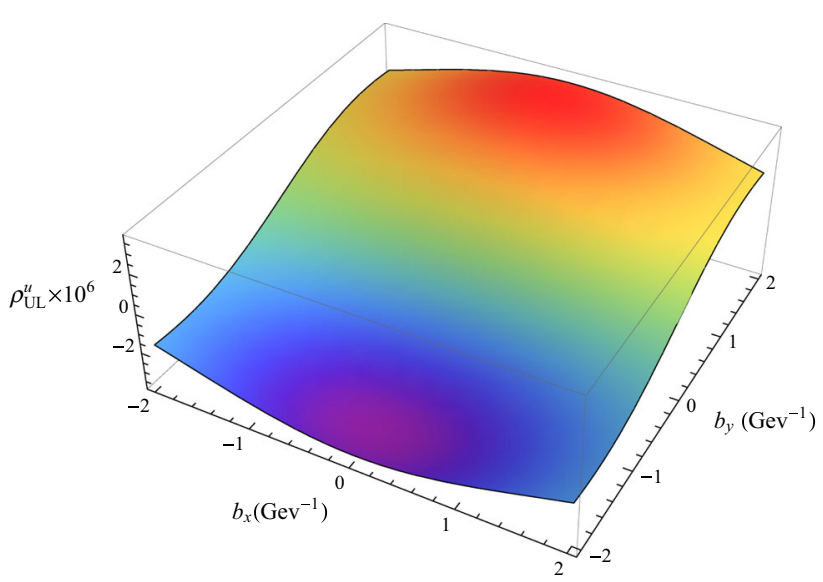

Fig. 17 Wigner distribution $\rho_{U L}^{u}\left(x, \mathbf{b}_{\perp}, \mathbf{k}_{\perp}\right)$ at $x=0.5, k_{x}=k_{y}=$ $0.5 \mathrm{GeV}$ 


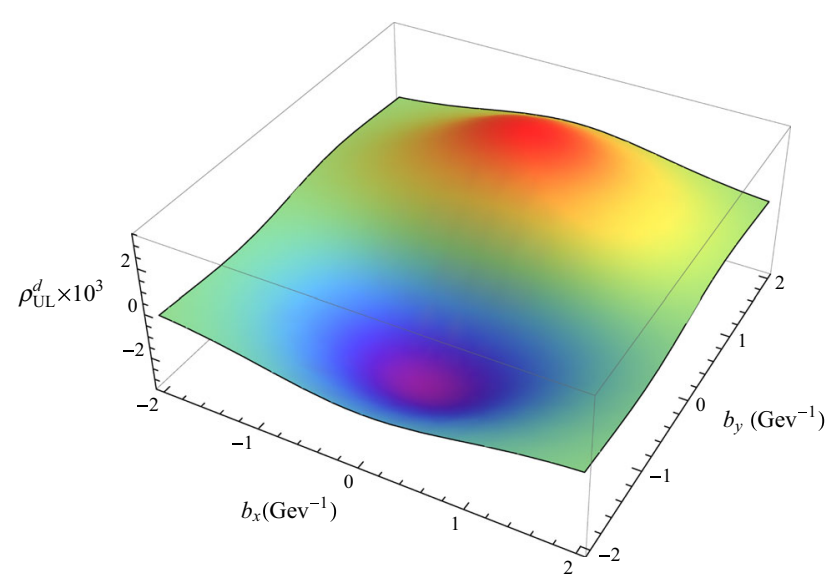

Fig. 18 Wigner distribution $\rho_{U L}^{d}\left(x, \mathbf{b}_{\perp}, \mathbf{k}_{\perp}\right)$ at $x=0.5, k_{x}=k_{y}=$ $0.5 \mathrm{GeV}$

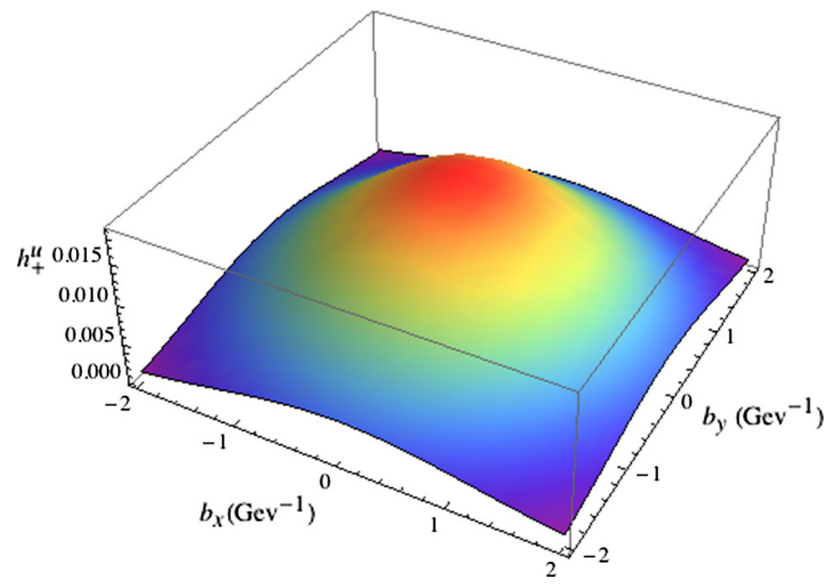

Fig. 19 Husimi distribution $h_{+}^{u}\left(x, \mathbf{b}_{\perp}, \mathbf{k}_{\perp}\right)$ at $x=0.5, l=1 \mathrm{GeV}^{-1}$, $k_{x}=k_{y}=0.5 \mathrm{GeV}$

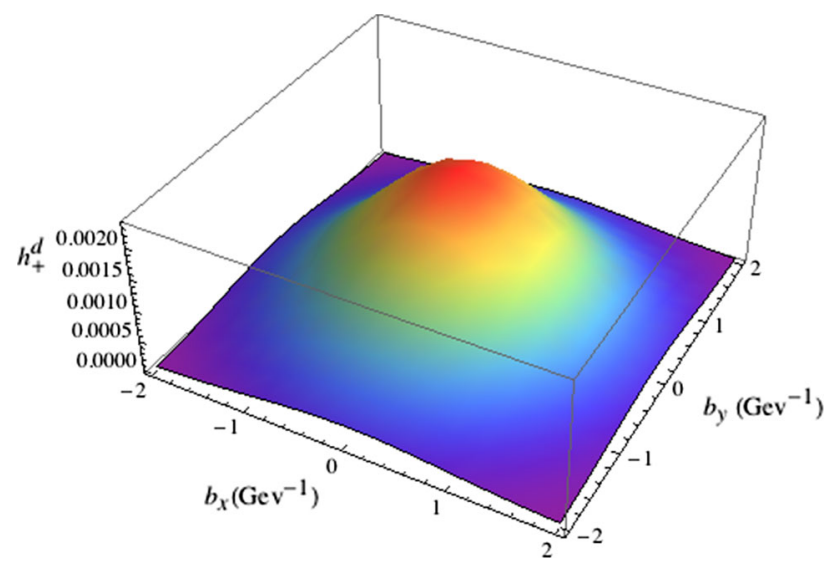

Fig. 20 Husimi distribution $h_{+}^{d}\left(x, \mathbf{b}_{\perp}, \mathbf{k}_{\perp}\right)$ at $x=0.5, l=1 \mathrm{GeV}^{-1}$, $k_{x}=k_{y}=0.5 \mathrm{GeV}$

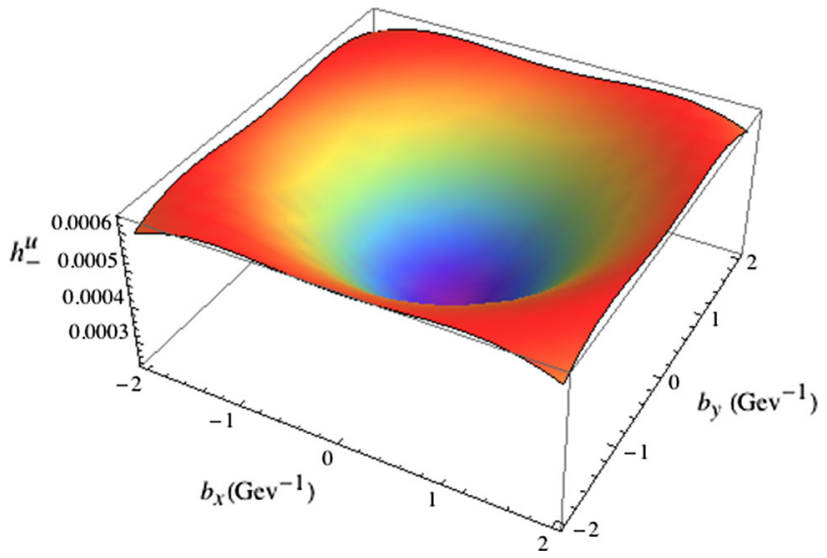

Fig. 21 Husimi distribution $h_{-}^{u}\left(x, \mathbf{b}_{\perp}, \mathbf{k}_{\perp}\right)$ at $x=0.5, l=1 \mathrm{GeV}^{-1}$, $k_{x}=k_{y}=0.5 \mathrm{GeV}$

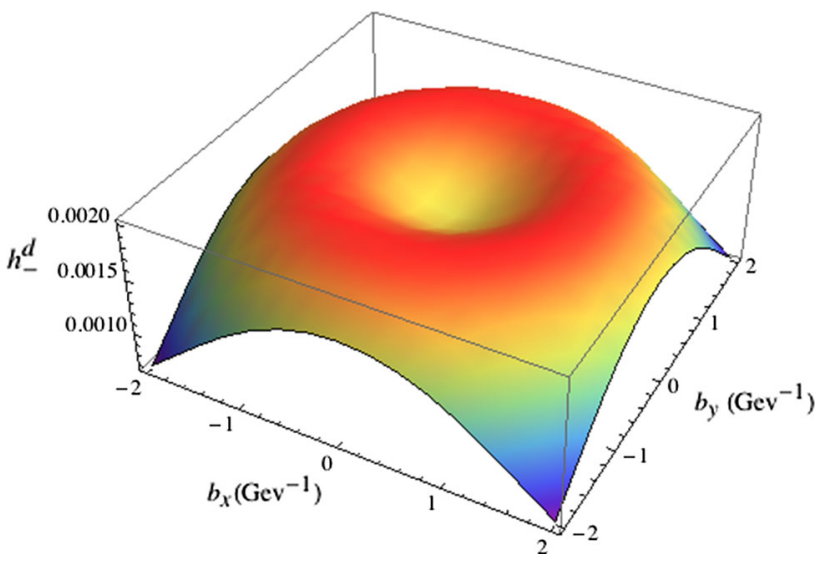

Fig. 22 Husimi distribution $h_{-}^{d}\left(x, \mathbf{b}_{\perp}, \mathbf{k}_{\perp}\right)$ at $x=0.5, l=1 \mathrm{GeV}^{-1}$, $k_{x}=k_{y}=0.5 \mathrm{GeV}$

\section{Conclusion}

We want to summarize the main result of our paper. In the quark-scalar diquark picture we propose LFWFs for the nucleon which analytically reproduce the quark PDFs in the nucleon at the initial scale $\mu \sim 1 \mathrm{GeV}$. Our LFWFs contain four longitudinal wave functions $D_{q}^{(i)}, q=u, d$ and $i=1,2$, depending on the $x$ variable, which are fixed from the analysis of nucleon form factors. Then we present a list of different types of nucleon parton distributions (TMDs, Wigner and Husimi distributions) in terms of the quark PDFs and the longitudinal functions $D_{q}^{(i)}$. Finally, we present the numerical analysis for the quark distributions in the nucleon, we also indicate selected results for the quark and nucleon form factors using a specific ansatz for the NLO helicity-independent and helicity-dependent parton distributions at $\mu_{\mathrm{NLO}}=0.40$ $\mathrm{GeV}^{2}[63,64]$. The resulting valence quark densities in the 
nucleon (e.g. TMDs) can be evolved to higher scales and can be compared to results for these quantities extracted in a data analysis.

Acknowledgements The authors thank Stan Brodsky, Lev Lipatov, Oleg Teryaev, Werner Vogelsang, and Marat Siddikov for useful discussions. This work was supported by the German Bundesministerium für Bildung und Forschung (BMBF) under Project 05P2015 ALICE at High Rate (BMBF-FSP 202): "Jet- and fragmentation processes at ALICE and the parton structure of nuclei and structure of heavy hadrons", by Tomsk State University Competitiveness Improvement Program and the Russian Federation program "Nauka" (Contract No. 0.1526.2015, 3854), by CONICYT (Chile) Research Project No. 80140097 and under Grants No. 7912010025,1140390 and PIA/Basal FB0821. VEL would like to thank Departamento de Física y Centro Científico Tecnológico de Valparaíso (CCTVal), Universidad Técnica Federico Santa María, Valparaíso, Chile for warm hospitality. In memory of my parents (VEL).

Open Access This article is distributed under the terms of the Creative Commons Attribution 4.0 International License (http://creativecomm ons.org/licenses/by/4.0/), which permits unrestricted use, distribution, and reproduction in any medium, provided you give appropriate credit to the original author(s) and the source, provide a link to the Creative Commons license, and indicate if changes were made.

Funded by SCOAP ${ }^{3}$.

\section{Appendix A: Wigner and Husimi parton distributions in the light-front quark model}

The Wigner distributions $\rho_{U U}^{q}\left(\mathbf{b}_{\perp}, \mathbf{k}_{\perp}, x\right)$ and $\omega_{U U}^{q}\left(\Delta_{\perp}\right.$, $\left.\mathbf{k}_{\perp}, x\right)$ read

$$
\begin{aligned}
& \rho_{U U}^{q}=\frac{1}{\pi^{2}(1-x)^{2}} \\
& \times\left[\frac{q_{v}(x)+\delta q_{v}(x)}{2} e^{-\frac{\mathbf{k}_{\perp}^{2}}{M_{N}^{2}} \alpha_{q}^{(1)}(x)} e^{-\mathbf{b}_{\perp}^{2} M_{N}^{2} \beta_{q}^{(1)}(x)}\right. \\
& +\frac{q_{v}(x)-\delta q_{v}(x)}{2} e^{-\frac{\mathbf{k}_{\perp}^{2}}{M_{N}^{2}} \alpha_{q}^{(2)}(x)} e^{-\mathbf{b}_{\perp}^{2} M_{N}^{2} \beta_{q}^{(2)}(x)} \\
& \left.\times\left[-1+\frac{\mathbf{k}_{\perp}^{2}}{M_{N}^{2}} \alpha_{q}^{(2)}(x)+\mathbf{b}_{\perp}^{2} M_{N}^{2} \beta_{q}^{(2)}(x)\right]\right], \\
& \rho_{L L}^{q}=\frac{1}{\pi^{2}(1-x)^{2}} \\
& \times\left[\frac{q_{v}(x)+\delta q_{v}(x)}{2} e^{-\frac{\mathbf{k}_{\perp}^{2}}{M_{N}^{2}} \alpha_{q}^{(1)}(x)} e^{-\mathbf{b}_{\perp}^{2} M_{N}^{2} \beta_{q}^{(1)}(x)}\right. \\
& -\frac{q_{v}(x)-\delta q_{v}(x)}{2} e^{-\frac{\mathbf{k}_{\perp}^{2}}{M_{N}^{2}} \alpha_{q}^{(2)}(x)} e^{-\mathbf{b}_{\perp}^{2} M_{N}^{2} \beta_{q}^{(2)}(x)} \\
& \left.\times\left[-1+\frac{\mathbf{k}_{\perp}^{2}}{M_{N}^{2}} \alpha_{q}^{(2)}(x)+\mathbf{b}_{\perp}^{2} M_{N}^{2} \beta_{q}^{(2)}(x)\right]\right], \\
& \rho_{U L}^{q}=-\rho_{L U}^{q}=\frac{1}{\pi^{2}(1-x)^{3}} \epsilon^{i j} \mathbf{k}_{\perp}^{i} \mathbf{b}_{\perp}^{j} \\
& \times\left[q_{v}(x)-\delta q_{v}(x)\right] e^{-\frac{\mathbf{k}_{\perp}^{2}}{M_{N}^{2}} \alpha_{q}^{(2)}(x)} e^{-\mathbf{b}_{\perp}^{2} M_{N}^{2} \beta_{q}^{(2)}(x)}
\end{aligned}
$$

and

$$
\begin{aligned}
\omega_{U U}^{q}\left(\Delta_{\perp}, \mathbf{k}_{\perp}, x\right)= & \frac{1}{\pi M_{N}^{2}}\left[\frac{q_{v}(x)+\delta q_{v}(x)}{2} \alpha_{q}^{(1)}(x)\right. \\
& \times e^{-\frac{\mathbf{k}_{\perp}^{2}+\frac{\Delta_{\perp}^{2}}{4}(1-x)^{2}}{M_{N}^{2}} \alpha_{q}^{(1)}(x)} \\
& +\frac{q_{v}(x)-\delta q_{v}(x)}{2}\left(\alpha_{q}^{(2)}(x)\right)^{2} \\
& \times \frac{\mathbf{k}_{\perp}^{2}+\frac{\Delta_{\perp}^{2}}{4}(1-x)^{2}}{M_{N}^{2}} \\
\omega_{L L}^{q}\left(\Delta_{\perp}, \mathbf{k}_{\perp}, x\right)= & \frac{1}{\pi M_{N}^{2}\left[\frac{q_{v}(x)+\delta q_{v}}{2}(x)\right.} \alpha_{q}^{(2)}(x) \\
& \times e^{-\frac{\mathbf{k}_{\perp}^{2}+\frac{\Delta_{\perp}^{2}}{4}(1-x)^{2}}{M_{N}^{2}} \alpha_{q}^{(1)}(x)}, \\
& -\frac{q_{v}(x)-\frac{\mathbf{k}_{\perp}^{2}}{4}(1-x)^{2}}{M_{N}^{2}} \alpha_{q}^{(2)}(x) \\
& e^{-\frac{\mathbf{k}_{\perp}^{2}+\frac{\Delta_{\perp}^{2}}{4}(1-x)^{2}}{M_{N}^{2}} \alpha_{q}^{(2)}(x)}, \\
& \times \frac{\mathbf{k}_{\perp}^{2}+\frac{\Delta_{\perp}^{2}}{4}(1-x)^{2}}{M_{N}^{2}}
\end{aligned}
$$

$$
\begin{aligned}
\omega_{U L}^{q}\left(\Delta_{\perp}, \mathbf{k}_{\perp}, x\right)= & -\omega_{L U}^{q}\left(\Delta_{\perp}, \mathbf{k}_{\perp}, x\right) \\
= & \frac{1}{\pi M_{N}^{4}} i \epsilon^{i j} \mathbf{k}_{\perp}^{i} \Delta_{\perp}^{j}\left[q_{v}(x)-\delta q_{v}(x)\right] \\
& \times e^{-\frac{\mathbf{k}_{\perp}^{2}}{M_{N}^{2}} \alpha_{q}^{(2)}(x)} e^{-\mathbf{b}_{\perp}^{2} M_{N}^{2} \beta_{q}^{(2)}(x)},
\end{aligned}
$$

where $\alpha_{q}^{(i)}(x)=D_{q}^{(i)}(x), \beta_{q}^{(i)}(x)=\frac{1}{(1-x)^{2} D_{q}^{(i)}(x)}, \epsilon^{12}=$ $-\epsilon^{21}=1$.

The integrals over the Wigner distributions are related to the TMDs, NPDs and PDFs by

$\int \mathrm{d}^{2} \mathbf{b}_{\perp} \rho_{U U}^{q}\left(\mathbf{b}_{\perp}, \mathbf{k}_{\perp}, x\right)=f_{1}^{q}\left(x, \mathbf{k}_{\perp}\right)$,
$\int \mathrm{d}^{2} \mathbf{b}_{\perp} \rho_{L L}^{q}\left(\mathbf{b}_{\perp}, \mathbf{k}_{\perp}, x\right)=g_{1 L}^{q}\left(x, \mathbf{k}_{\perp}\right)$,

$\int \mathrm{d}^{2} \mathbf{b}_{\perp} \omega_{U U}^{q}\left(\Delta_{\perp}, \mathbf{k}_{\perp}, x\right)=\mathcal{H}\left(x, 0, \Delta_{\perp}^{2}\right)$,

$\int \mathrm{d}^{2} \mathbf{b}_{\perp} \omega_{L L}^{q}\left(\Delta_{\perp}, \mathbf{k}_{\perp}, x\right)=\tilde{\mathcal{H}}\left(x, 0, \Delta_{\perp}^{2}\right)$,

and

$\int \mathrm{d}^{2} \mathbf{k}_{\perp} \mathrm{d}^{2} \mathbf{b}_{\perp} \rho_{U U}^{q}\left(x, \mathbf{k}_{\perp}, \mathbf{b}_{\perp}\right)=q_{v}(x)=f_{1}^{q_{v}}(x)$,

$\int \mathrm{d}^{2} \mathbf{k}_{\perp} \mathrm{d}^{2} \mathbf{b}_{\perp} \rho_{L L}^{q}\left(x, \mathbf{k}_{\perp}, \mathbf{b}_{\perp}\right)=\delta q_{v}(x)=g_{1 L}^{q_{v}}(x)$, 
and

$$
\begin{aligned}
& \int_{0}^{1} \mathrm{~d} x \int \mathrm{d}^{2} \mathbf{k}_{\perp} \mathrm{d}^{2} \mathbf{b}_{\perp} \rho_{U U}^{q}\left(x, \mathbf{k}_{\perp}, \mathbf{b}_{\perp}\right)=n_{q}, \\
& \int_{0}^{1} \mathrm{~d} x \int \mathrm{d}^{2} \mathbf{k}_{\perp} \mathrm{d}^{2} \mathbf{b}_{\perp} \rho_{L L}^{q}\left(x, \mathbf{k}_{\perp}, \mathbf{b}_{\perp}\right)=g_{A}^{q}, \\
& \int_{0}^{1} \mathrm{~d} x \int \mathrm{d}^{2} \mathbf{k}_{\perp} \mathrm{d}^{2} \mathbf{b}_{\perp} \rho_{U L}^{q}\left(x, \mathbf{k}_{\perp}, \mathbf{b}_{\perp}\right) \\
& =\int_{0}^{1} \mathrm{~d} x \int \mathrm{d}^{2} \mathbf{k}_{\perp} \mathrm{d}^{2} \mathbf{b}_{\perp} \rho_{L U}^{q}\left(x, \mathbf{k}_{\perp}, \mathbf{b}_{\perp}\right)=0 .
\end{aligned}
$$

The Husimi parton distributions are given by

$$
\begin{aligned}
& h_{U U}^{q}\left(x, \mathbf{b}_{\perp}, \mathbf{k}_{\perp}\right)+h_{L L}^{q}\left(x, \mathbf{b}_{\perp}, \mathbf{k}_{\perp}\right) \\
&= \frac{M_{N}^{2} l^{2}}{\pi^{2}}\left(q_{v}(x)+\delta q_{v}(x)\right) \rho_{q}^{(1)}(x) \sigma_{q}^{(1)}(x) \\
& \quad \times e^{-\mathbf{k}_{\perp}^{2} l^{2} \rho_{q}^{(1)}(x)} e^{-\mathbf{b}_{\perp}^{2} M_{N}^{2} \sigma_{q}^{(1)}(x)}, \\
& h_{U U}^{q}\left(x, \mathbf{b}_{\perp}, \mathbf{k}_{\perp}\right)-h_{L L}^{q}\left(x, \mathbf{b}_{\perp}, \mathbf{k}_{\perp}\right) \\
&=\frac{M_{N}^{2} l^{2}}{\pi^{2}}\left(q_{v}(x)-\delta q_{v}(x)\right) \rho_{q}^{(2)}(x) \sigma_{q}^{(2)}(x) \\
& \quad \times e^{-\mathbf{k}_{\perp}^{2} l^{2} \rho_{q}^{(2)}(x)} e^{-\mathbf{b}_{\perp}^{2} M_{N}^{2} \sigma_{q}^{(2)}(x)} \\
& \quad \times\left[1+\frac{M_{N}^{2} l^{2}}{D_{q}^{(2)}(x)} \rho_{q}^{(2)}(x)\left(\mathbf{k}_{\perp}^{2} l^{2} \rho_{q}^{(2)}(x)-1\right)\right. \\
&\left.\quad+D_{q}^{(2)}(x) \sigma_{q}^{(2)}(x)(1-x)^{2}\left(\mathbf{b}_{\perp}^{2} M_{N}^{2} \sigma_{q}^{(2)}(x)-1\right)\right], \\
& \quad \times h_{U L}^{q}\left(x, \mathbf{b}_{\perp}, \mathbf{k}_{\perp}\right)=-h_{L U}^{q}\left(\mathbf{b}_{\perp}, \mathbf{k}_{\perp}, x\right) \\
&=\frac{1}{\pi^{2}} \epsilon^{i j} \mathbf{k}_{\perp}^{i} \mathbf{b}_{\perp}^{j} \\
& \quad \times\left[q_{v}(x)-\delta q_{v}(x)\right](1-x) M_{N}^{4} l^{4}\left[\rho_{q}^{(2)}(x) \sigma_{q}^{(2)}(x)\right]^{2} \\
& \quad \times e^{-\mathbf{k}_{\perp}^{2} l^{2} \rho_{q}^{(2)}(x)} e^{-\mathbf{b}_{\perp}^{2} M_{N}^{2} \sigma_{q}^{(2)}(x)},
\end{aligned}
$$

where

$\rho_{q}^{(i)}(x)=\frac{D_{q}^{(i)}(x)}{M_{N}^{2} l^{2}+D_{q}^{(i)}(x)}, \quad \sigma_{q}^{(i)}(x)=\frac{1}{M_{N}^{2} l^{2}+D_{q}^{(i)}(x)(1-x)^{2}}$.

The expressions for the entropies of the nucleon $S_{ \pm}^{q}(x)$ are given by

$$
\begin{aligned}
& S_{+}^{q}(x)=\left(q_{v}(x)+\delta q_{v}(x)\right)\left[1-\frac{1}{2} \log \left(\frac{q_{v}(x)+\delta q_{v}(x)}{2 \pi^{2}}\right)\right], \\
& S_{-}^{q}(x)=\left(q_{v}(x)-\delta q_{v}(x)\right)\left[1-\frac{1}{2} \log \left(\frac{q_{v}(x)-\delta q_{v}(x)}{2 \pi^{2}}\right)\right.
\end{aligned}
$$

$$
\begin{aligned}
& -\frac{1}{2} \log (B)-\frac{A_{1}+A_{2}}{4} \\
& \times\left(A_{1} \int_{0}^{\infty} \frac{d t e^{-t}}{A_{1} t+B}+A_{2} \int_{0}^{\infty} \frac{d t e^{-t}}{A_{2} t+B}\right) \\
& \left.-\frac{A_{1}^{2}+A_{2}^{2}}{4} \int_{0}^{\infty} \int_{0}^{\infty} \frac{d t_{1} d t_{2} e^{-t_{1}-t_{2}}}{A_{1} t_{1}+A_{2} t_{2}+B}\right],
\end{aligned}
$$

where

$$
\begin{aligned}
& A_{1}=\frac{D_{q}^{(2)}(x)(1-x)^{2}}{M_{N}^{2} l^{2}+D_{q}^{(2)}(x)(1-x)^{2}} \\
& A_{2}=\frac{M_{N}^{2} l^{2}}{M_{N}^{2} l^{2}+D_{q}^{(2)}(x)}, B=1-A_{1}-A_{2} .
\end{aligned}
$$

\section{References}

1. T. Gutsche, V.E. Lyubovitskij, I. Schmidt, A. Vega, Phys. Rev. D 89, 054033 (2014)

2. T. Gutsche, V.E. Lyubovitskij, I. Schmidt, A. Vega, Phys. Rev. D 91, 054028 (2015)

3. S.J. Brodsky, G.R. Farrar, Phys. Rev. Lett. 31, 1153 (1973)

4. V.A. Matveev, R.M. Muradian, A.N. Tavkhelidze, Lett. Nuovo Cim. 7, 719 (1973)

5. V.N. Gribov, L.N. Lipatov, Sov. J. Nucl. Phys. 15, 438 (1972) [Yad. Fiz. 15, 781 (1972)]

6. V.N. Gribov, L.N. Lipatov, Sov. J. Nucl. Phys. 15, 675 (1972) [Yad.Fiz. 15, 1218 (1972)]

7. G. Altarelli, G. Parisi, Nucl. Phys. B 126, 298 (1977)

8. Y.L. Dokshitzer, Sov. Phys. JETP 46, 641 (1977) [Zh. Eksp. Teor. Fiz. 73, 1216 (1977)]

9. T. Gutsche, V.E. Lyubovitskij, I. Schmidt, A. Vega, J. Phys. G 42, 095005 (2015)

10. S.J. Brodsky, T. Huang, G.P. Lepage, Springer Tracts Mod. Phys. 100, 81 (1982)

11. S.J. Brodsky, D.S. Hwang, Nucl. Phys. B 543, 239 (1999)

12. S.J. Brodsky, D.S. Hwang, B.-Q. Ma, I. Schmidt, Nucl. Phys. B 593, $311(2001)$

13. S.J. Brodsky, M. Diehl, D.S. Hwang, Nucl. Phys. B 596, 99 (2001)

14. B.Q. Ma, I. Schmidt, J. Soffer, Phys. Lett. B 441, 461 (1998)

15. B.Q. Ma, D. Qing, I. Schmidt, Phys. Rev. C 65, 035205 (2002)

16. B. Pasquini, S. Cazzaniga, S. Boffi, Phys. Rev. D 78, 034025 (2008)

17. A. Bacchetta, F. Conti, M. Radici, Phys. Rev. D 78, 074010 (2008)

18. J. She, J. Zhu, B.Q. Ma, Phys. Rev. D 79, 054008 (2009)

19. A. Courtoy, S. Scopetta, V. Vento, Phys. Rev. D 80, 074032 (2009)

20. Z. Lu, I. Schmidt, Phys. Rev. D 82, 094005 (2010)

21. C. Lorce, B. Pasquini, Phys. Rev. D 84, 014015 (2011)

22. C. Lorce, B. Pasquini, X. Xiong, F. Yuan, Phys. Rev. D 85, 114006 (2012)

23. S.S. Chabysheva, J.R. Hiller, Ann. Phys. 337, 143 (2013)

24. L. Gamberg, Z.B. Kang, I. Vitev, H. Xing, Phys. Lett. B 743, 112 (2015)

25. T. Liu, B.Q. Ma, Phys. Rev. D 91, 034019 (2015)

26. T. Maji, C. Mondal, D. Chakrabarti, O.V. Teryaev, JHEP 1601, 165 (2016)

27. D. Chakrabarti, T. Maji, C. Mondal, A. Mukherjee, Eur. Phys. J. C 76, 409 (2016)

28. S.J. Brodsky, G.F. de Teramond, Phys. Rev. D 77, 056007 (2008)

29. G.F. de Teramond, S.J. Brodsky, AIP Conf. Proc. 1432, 168 (2012) 
30. S.J. Brodsky, G.F. de Teramond, AIP Conf. Proc. 1388, 22 (2011)

31. S.J. Brodsky, F.-G. Cao, G.F. de Teramond, Phys. Rev. D 84, $075012(2011)$

32. Z. Abidin, C.E. Carlson, Phys. Rev. D 79, 115003 (2009)

33. A. Vega, I. Schmidt, T. Branz, T. Gutsche, V.E. Lyubovitskij, Phys. Rev. D 80, 055014 (2009)

34. T. Branz, T. Gutsche, V.E. Lyubovitskij, I. Schmidt, A. Vega, Phys. Rev. D 82, 074022 (2010)

35. T. Gutsche, V.E. Lyubovitskij, I. Schmidt, A. Vega, Phys. Rev. D 87, 056001 (2013)

36. T. Gutsche, V.E. Lyubovitskij, I. Schmidt, A. Vega, Phys. Rev. D 85, 076003 (2012)

37. T. Gutsche, V.E. Lyubovitskij, I. Schmidt, A. Vega, Phys. Rev. D 86, 036007 (2012)

38. T. Gutsche, V.E. Lyubovitskij, I. Schmidt, A. Vega, Phys. Rev. D 87, 016017 (2013)

39. A.V. Radyushkin, Phys. Rev. D 58, 114008 (1998)

40. S.J. Brodsky, S.D. Drell, Phys. Rev. D 22, 2236 (1980)

41. A. Bacchetta, A. Courtoy, M. Radici, JHEP 1303, 119 (2013)

42. M. Anselmino, M. Boglione, U. D'Alesio, S. Melis, F. Murgia, A. Prokudin, Phys. Rev. D 87, 094019 (2013)

43. A. Signori, A. Bacchetta, M. Radici, G. Schnell, JHEP 1311, 194 (2013)

44. M. Radici, A. Courtoy, A. Bacchetta, M. Guagnelli, JHEP 1505, $123(2015)$

45. Z.B. Kang, A. Prokudin, P. Sun, F. Yuan, Phys. Rev. D 93, 014009 (2016)

46. C. Lorce, B. Pasquini, Phys. Rev. D 84, 034039 (2011)

47. J. Soffer, Phys. Rev. Lett. 74, 1292 (1995)

48. A. Bacchetta, M. Boglione, A. Henneman, P.J. Mulders, Phys. Rev. Lett. 85, 712 (2000)
49. H. Avakian, A.V. Efremov, P. Schweitzer, F. Yuan, Phys. Rev. D 81, 074035 (2010)

50. A.V. Efremov, P. Schweitzer, O.V. Teryaev, P. Zavada, Phys. Rev. D 80, 014021 (2009)

51. X.D. Ji, Phys. Rev. Lett. 91, 062001 (2003)

52. A.V. Belitsky, X.D. Ji, F. Yuan, Phys. Rev. D 69, 074014 (2004)

53. S. Meissner, A. Metz, M. Schlegel, K. Goeke, JHEP 0808, 038 (2008)

54. S. Meissner, A. Metz, M. Schlegel, JHEP 0908, 056 (2009)

55. X.D. Ji, Phys. Rev. Lett. 78, 610 (1997)

56. H. Avakian, A.V. Efremov, P. Schweitzer, F. Yuan, Phys. Rev. D 78, $114024(2008)$

57. H. Avakian, A.V. Efremov, P. Schweitzer, O.V. Teryaev, F. Yuan, P. Zavada, Mod. Phys. Lett. A 24, 2995 (2009)

58. M. Burkardt, B.C. Hikmat, Phys. Rev. D 79, 071501 (2009)

59. K. Kanazawa, C. Lorc, A. Metz, B. Pasquini, M. Schlegel, Phys. Rev. D 90, 014028 (2014)

60. Y. Hagiwara, Y. Hatta, Nucl. Phys. A 940, 158 (2015)

61. Y. Hatta, Y. Hagiwara, EPJ Web Conf. 112, 01010 (2016)

62. A. Wehrl, Rev. Mod. Phys. 50, 221 (1978)

63. M. Gluck, E. Reya, A. Vogt, Eur. Phys. J. C 5, 461 (1998)

64. M. Gluck, E. Reya, M. Stratmann, W. Vogelsang, Phys. Rev. D 63, 094005 (2001)

65. M. Diehl, P. Kroll, Eur. Phys. J. C 73, 2397 (2013)

66. M. Diehl, Nucl. Phys. Proc. Suppl. 161, 49 (2006)

67. G.D. Cates, C.W. de Jager, S. Riordan, B. Wojtsekhowski, Phys. Rev. Lett. 106, 252003 (2011)

68. M. Guidal, M.V. Polyakov, A.V. Radyushkin, M. Vanderhaeghen, Phys. Rev. D 72, 054013 (2005)

69. A. Mukherjee, S. Nair, V.K. Ojha, Phys. Rev. D 90, 014024 (2014) 